\title{
STRUCTURE-ACTIVITY RELATIONSHIPS IN CHEMICALLY MODIFIED COUMERMYCIN
}

\author{
J. G. KeIL, J. C. Godfrey, M. J. Cron, I. R. HoOper, \\ D. E. Nettleton, K. E. Price and H. Schmitz \\ Research Division, Bristol Laboratories, \\ Division of Bristol-Myers Company, Syracuse, New York, USA
}

\begin{abstract}
Coumermycin $A_{1}$ was early recognized as a potentially useful antibiotic because of its very good spectrum of activity against gram-positive organisms (particularly against staphylococci), in addition to appreciable activity against a variety of gram-negative bacteria. However, in spite of its excellent in vitro activity, the insoluble character of coumermycin $A_{1}$ made parenteral administration unsatisfactory. Poor oral absorption was also observed.

Because of these shortcomings, coumermycin $A_{1}$ was chemically modified either by an acyl interchange reaction or by direct condensation with 3amino-4-hydroxy-8-methyl-7-[3-O-(5-methyl-2-pyrrolylcarbonyl)noviosyloxy] coumarin (PNC-amine), which was produced by chemical degradation of coumermycin $A_{1}$. A large number of semisynthetic derivatives were so prepared, and interesting structure-activity patterns will be discussed. A number of the new semisynthetic derivatives possessed markedly improved oral absorbability in mice. This improvement in some cases was $>25$ fold over the parent compound and more than compensated for the lower antibacterial potency possessed by most of the derivatives. Further, some of the more active compounds showed a significant improvement in the in vivo antistaphylococcal therapeutic effect, and marked improvement in oral efficacy in experimental D. pneumoniae and Streptococcus pyogenes mouse infections.

Compounds demonstrating the broadest spectrum of antibacterial activity were members of the 3-substituted-4-hydroxybenzoic acid class.

Among the aliphatic derivatives, 3-isobutyramido-4-hydroxy-8-methyl-7[3-O-(5-methyl-2-pyrrolylcarbonyl)noviosyloxy]coumarin (designated as BLC43) was by far the most interesting. Characterized by its overall trend toward improved in vitro activity, and its significant in vivo activity, this compound was selected for extensive pre-clinical evaluation. The results of these investigations will be discussed.
\end{abstract}

The complete structure of coumermycin $\mathrm{A}_{1}$ (Figure $1, \mathbf{R}=\mathrm{CH}_{3}$ ) was first disclosed in a US patent to Kawaguchi, Okanishi, and Miyaki in $1965^{1}$. Further, the importance of coumermycin $A_{1}$ as a powerful and apparently non-toxic antibiotic with an excellent spectrum of activity against grampositive organisms, as well as considerable activity against a variety of 


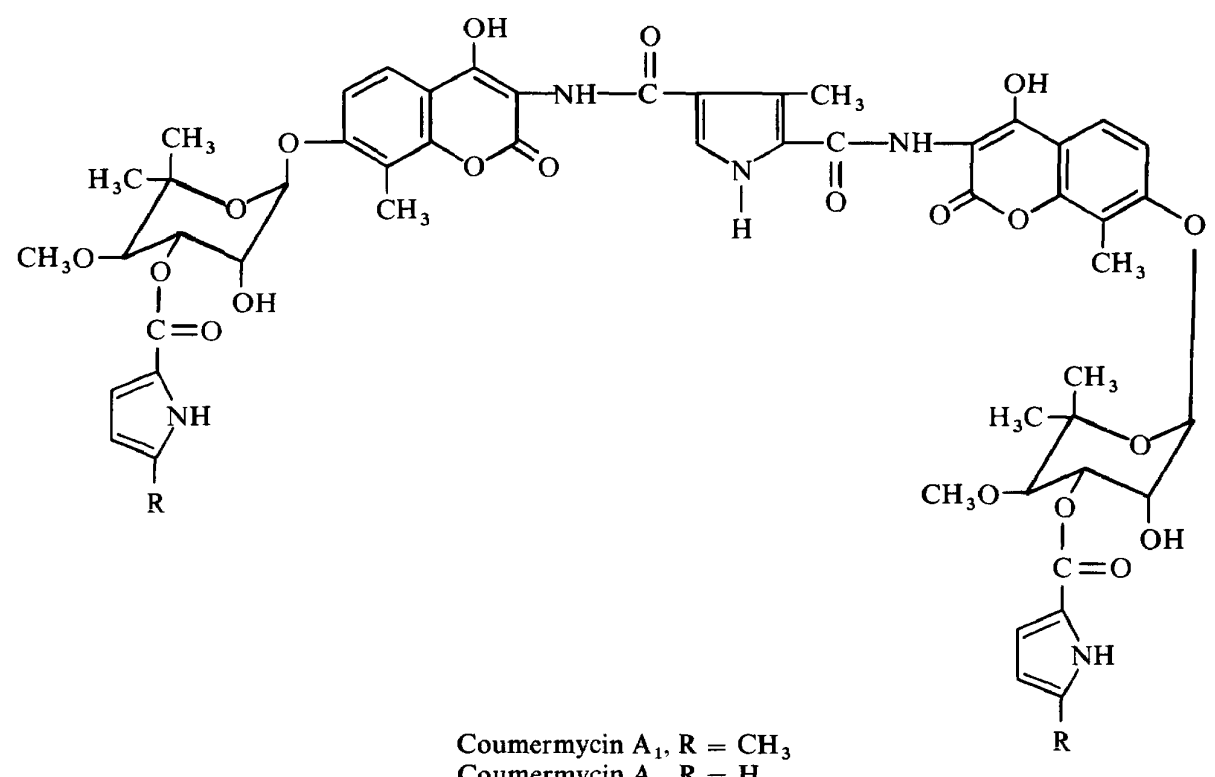

Figure 1. (Reproduced by permission of Journal of Antibiotics, Tokyo)

gram-negative bacteria, was immediately recognized ${ }^{2}$. Additional information on the structure of coumermycin $A_{1}$, as well as on the structure of coumermycin $\mathrm{A}_{2}$ (Figure $1, \mathbf{R}=\mathbf{H}$ ). followed in later publications by Dr Kawaguchi.

It should be recognized that, during this same period, workers at HoffmannLaRoche Corporation were also working on the same antibiotic complex and published its isolation from fermentation broths as well as a total synthesis in $1966^{3}$. Coumermycin $A_{2}$ differs from coumermycin $A_{1}$ only in the absence of the two 5-methyl groups in the terminal 2-pyrrolecarboxylic ester moieties. This small difference has a very significant effect on the antimicrobial activities of the two compounds. This dramatic difference in the in vitro activities of coumermycin $\mathrm{A}_{1}$ and $\mathrm{A}_{2}$ is indicated in Table 1.

Maximal activity for both antibiotics is exhibited against staphylococci, but coumermycin $A_{1}$ is 60 times more active than $A_{2}$. The extreme inactivating effect of serum on these antibiotics is also illustrated in Table 1. Serum ( 50 per cent in Heart Infusion Broth) depresses the activity of coumermycin $A_{1}$ to $1 / 400$ th of its activity in the absence of serum, and has a similar effect on coumermycin $\mathbf{A}_{2}$. That the very high anti-staphylococcal activity of coumermycin $A_{1}$ can still be achieved in an experimental mouse infection, in spite of extreme serum inactivation, is indicated in Table 2.

In spite of its excellent in vivo activity, the high degree of insolubility of coumermycin $A_{1}$, even as the sodium salt, made parenteral administration unsatisfactory. Poor oral absorption was also observed. These findings 


\section{CHEMICALLY MODIFIED COUMERMYCIN}

Table 1. Coumermycin antimicrobial spectra. MIC's in $\mu \mathrm{g} / \mathrm{ml}$, Heart Infusion Broth

\begin{tabular}{|c|c|c|}
\hline Organism & Coumermycin $A_{1}$ & Coumermycin $\mathrm{A}_{2}$ \\
\hline Staphylococcus aureus Smith & $0.004 \leftarrow(62 \times) \rightarrow$ & 0.25 \\
\hline Staphylococcus aureus Smith + serum & $1.6(400 \times)$ & $50(200 \times)$ \\
\hline Streptococcus pyogenes & 0.062 & 0.5 \\
\hline Bacillus subtilis & 6.3 & 12.5 \\
\hline Escherichia coli & 12.5 & $>100$ \\
\hline Klebsiella pneumoniae & 6.3 & 12.5 \\
\hline Proteus morganii & 6.2 & 25 \\
\hline Proteus mirabilis & 12.5 & $>100$ \\
\hline Proteus vulgaris & 3.1 & $>100$ \\
\hline Pseudomonas aeruginosa 8206A & 12.5 & $>100$ \\
\hline Pseudomonas aeruginosa Yale & 25 & $>100$ \\
\hline Salmonella enteritidis & 12.5 & 50 \\
\hline Salmonella typhi & 12.5 & $>100$ \\
\hline
\end{tabular}

coupled with the extreme serum inactivation of coumermycin $A_{1}$ resulted in our initiating. an extensive programme directed at modification of the coumermycin nucleus. The purposes were fourfold:

1. Reduction of the high level of serum inactivation.

2. Reduction of irritation liability.

3. Preservation of high activity.

4. Improvement of oral absorbability.

The close structural similarity between coumermycin $A_{1}$ and novobiocin, an antibiotic which is well absorbed orally, was immediately recognized (see Figure 2).

The relationship between the sugar (noviose, $N$ ) and coumarin (2-Ramino-4-hydroxy-7-glycosidyl-8-methyl-coumarin, $C$ ) moieties in novobiocin is the same as is present in coumermycin $A_{1}$. However, novobiocin has a simple carbamoyl function, - $\mathrm{CONH}_{2}$, in place of the 5-methylpyrrolyl2-carbonyl group $(P)$, and a 4-hydroxy-3-(3-methyl-2-butenyl)-benzamide in place of the 3-methylpyrrole-2,4-dicarboxamide CNP (D-NH-CNP) of coumermycin $\mathrm{A}_{1}$. Displacement of the 3-methylpyrrole-2,4-dicarboxamide function of coumermycin $A_{1}$ with simpler amides appeared to be the most logical starting point for obtaining structures which would hopefully have many of the desirable features of coumermycin $A_{1}$, but which would have increased solubility and be more readily absorbed via the oral route. A direct biosynthetic approach for producing coumermycin analogues by the

Table 2. Coumermycin activity in vivo

\begin{tabular}{lccccc}
\hline $\begin{array}{c}\text { Test } \\
\text { animal }\end{array}$ & Organism & $\begin{array}{c}\text { Treatment } \\
\text { route }\end{array}$ & $\begin{array}{c}\text { Time of } \\
\text { treatment }\end{array}$ & $\begin{array}{c}\mathrm{CD}_{50}, \mathrm{mg} / \mathrm{kg} \\
\text { Coumermycin } \mathrm{A}_{1}\end{array}$ & $\mathrm{~A}_{2}$ \\
\hline mouse & Staph. aureus (Smith) & IM & $\begin{array}{l}\text { immediately after } \\
\text { challenge }\end{array}$ & 0.37 & 4.5 \\
mouse & Staph.aureus (Smith) & oral & $\begin{array}{l}\text { immediately after } \\
\text { challenge }\end{array}$ & 2.7 to 15 & \\
\hline
\end{tabular}




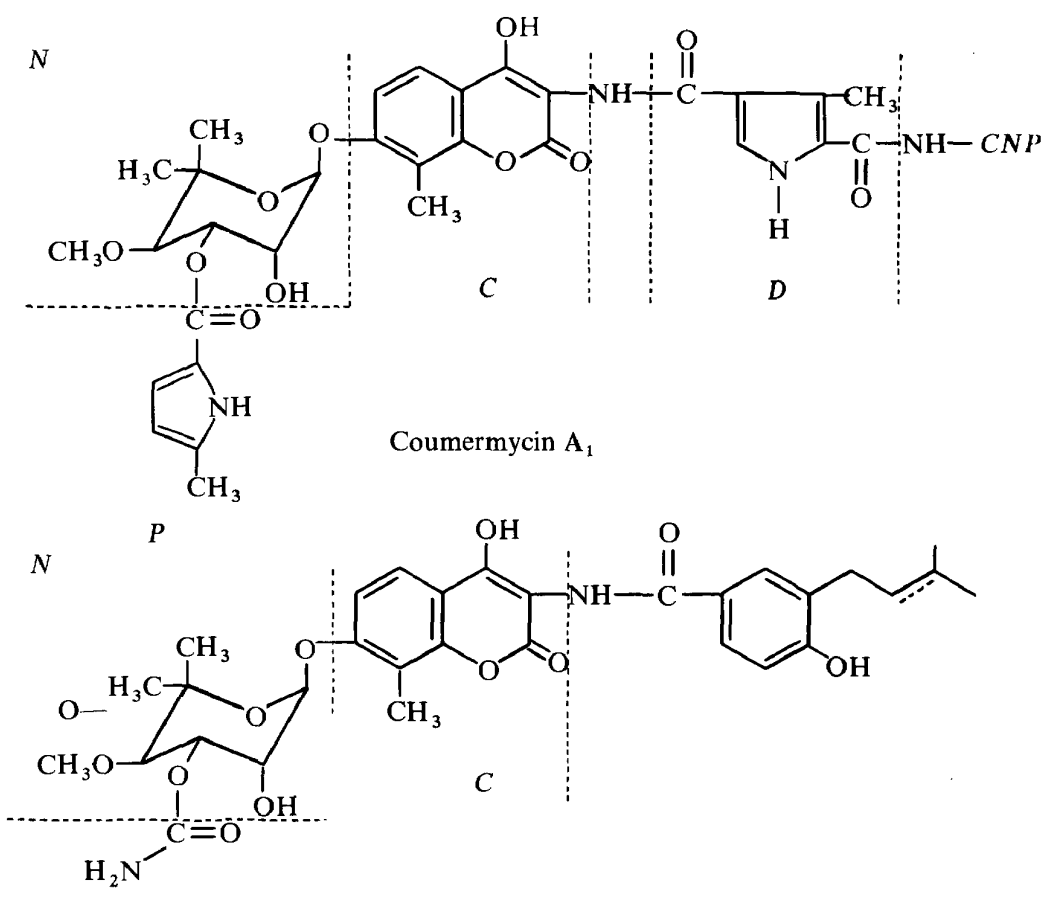

Novobiocin

(Dihydronovobiocin)

Figure 2

addition of a variety of aromatic acids to our coumermycin fermentations at various times, failed to afford any new bioactive materials detectable by standard chromatographic procedures. Walton and co-workers ${ }^{4}$ were able to accomplish this very successfully in the case of novobiocin.

Some interesting information on the displacement of the 3-methylpyrrole2,4-dicarboxamide function ( $D$ moiety) of coumermycin $A_{1}$ was obtained from the work of Hinman et al. ${ }^{5}$. These workers found that treatment of novobiocin with hot acetic anhydride results in displacement of the benzoyl moiety to give 4-acetoxy-3-(3-methyl-2-butenyl)benzoic acid and the formation of a 2-methyloxazole fused to the coumarin ring (Figure 3).<smiles></smiles>

Figure 3. (Reproduced by permission of Journal of Antibiotics, Tokyo) 
CHEMICALLY MODIFIED COUMERMYCIN

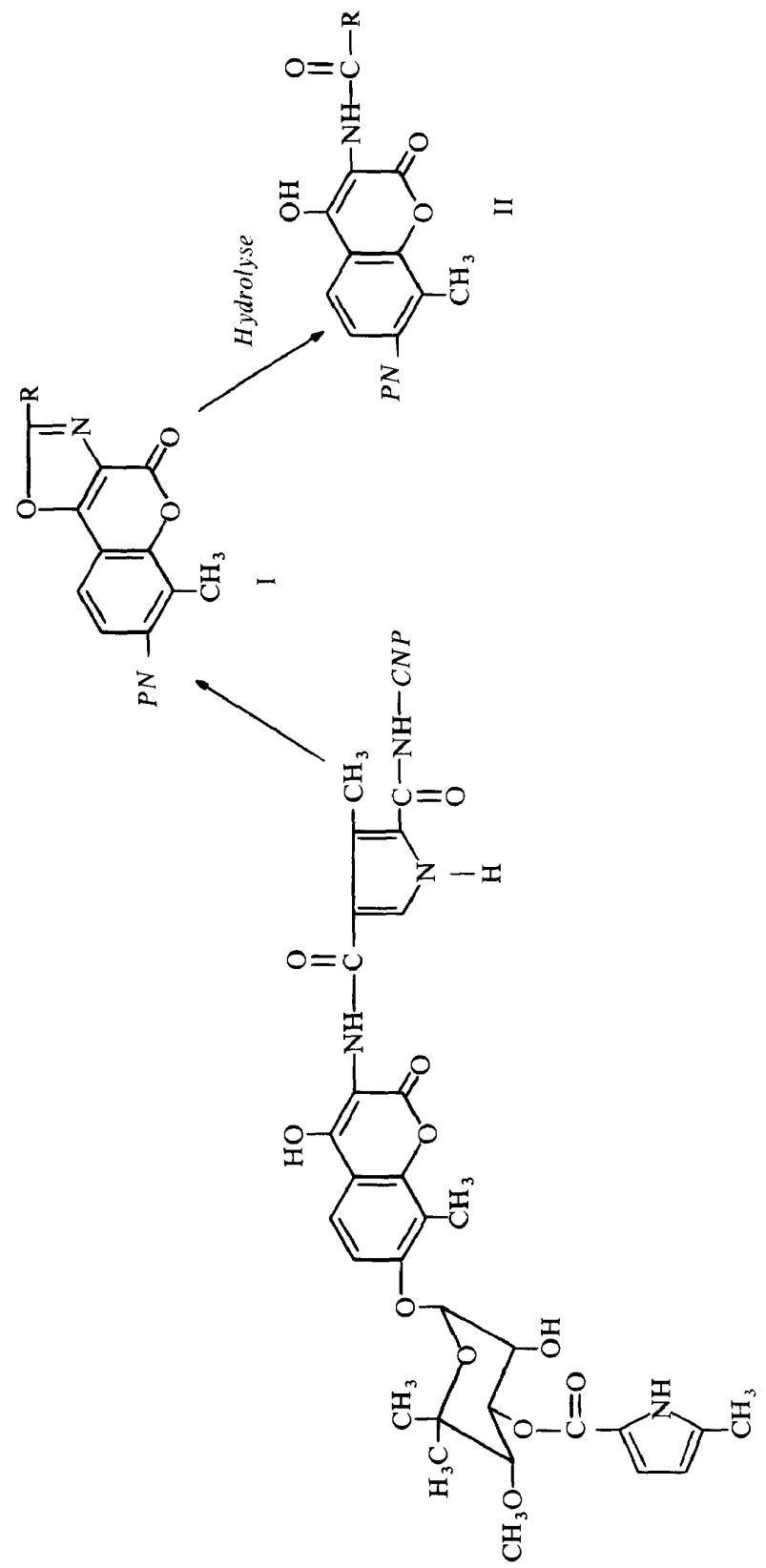

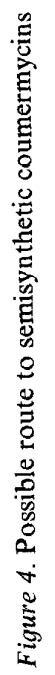

575 


\section{J. G. KEIL et al.}

Application of this information to the coumermycin problem seemed like a sound, logical starting point. Initial efforts were directed toward finding conditions for the acetylation which would result either in direct displacement of the $D$ moiety, or the formation of an analogous oxazole (Compound I of Figure 4), or possibly finding suitable hydrolytic conditions for converting I to new derivatives II. The more practical approach appeared to be the first-mentioned route, but this resulted in a derivative in which the 2-hydroxyl of the noviose was blocked with an acetyl group. Compounds of this type were devoid of antibiotic activity. Thus in order to avoid acylation of the free hydroxyls of the noviose moieties, and to prevent possible 5-methylpyrrolecarboxylate migration, it was essential to find suitable hydroxyl blocking groups. The tetrahydropyranyl group proved most versatile for our purposes.

Treatment of coumermycin $A_{1}$ in a mixture of tetrahydrofuran and dihydropyran containing a catalytic amount of $p$-toluenesulphonic acid monohydrate with stirring at $25^{\circ} \mathrm{C}$ gave a high yield of the desired $2^{\prime}, 2^{\prime}-O, O$ ditetrahydropyranylcoumermycin $\mathrm{A}_{1}{ }^{6,7}$ (III, Figure 5). Coumermycin $\mathrm{A}_{1}$

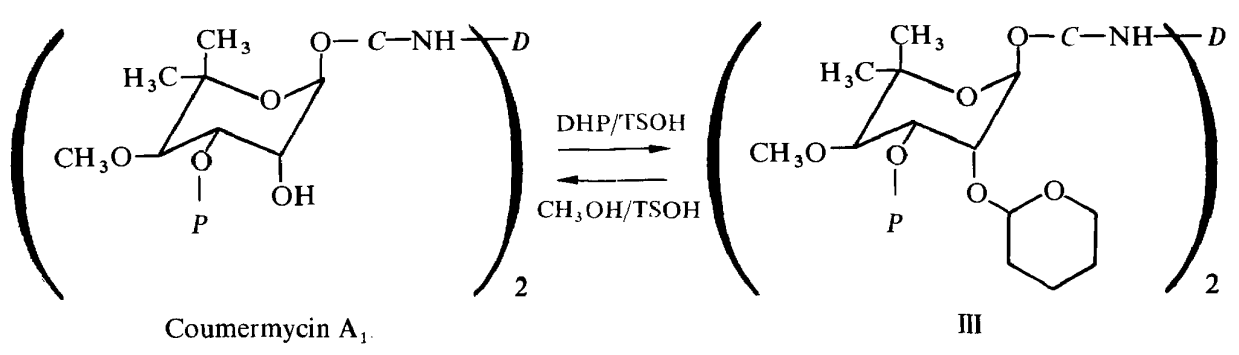

Figure 5. Ditetrahydropyranyl coumermycin $\mathbf{A}_{1}$<smiles>[R]C(=O)Nc1ccccc1OC([R])=O</smiles>

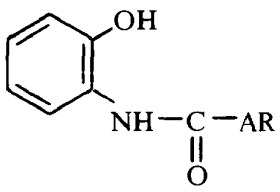<smiles>[R]C(=O)Nc1ccccc1OC([R])=O</smiles>

IVa, $\begin{aligned} \mathrm{R} & =\text { alkyl } \\ \mathrm{AR} & =\mathrm{C}_{6} \mathrm{H}_{5} \\ \text { IVb, } \mathrm{R} & =\mathrm{C}_{6} \mathrm{H}_{5} \\ \mathrm{AR} & =\alpha \text {-naphthyl }\end{aligned}$

$\mathrm{Va}, \mathrm{AR}=\mathrm{C}_{6} \mathrm{H}_{5}$

$\mathrm{Vb}, \mathrm{AR}=\alpha$-naphthyl

$$
\text { VIa. } \begin{aligned}
\mathrm{R} & =\text { alkyl } \\
\mathrm{AR} & =\mathrm{C}_{6} \mathrm{H}_{5} \\
\text { VIb, } \mathrm{R} & =\mathrm{C}_{6} \mathrm{H}_{5} \\
\mathrm{AR} & =\alpha \text {-naphthyl }
\end{aligned}
$$

Figure 6. (Reproduced by permission of Journal of Antibiotics, Tokyo) 


\section{CHEMICALLY MODIFIED COUMERMYCIN}

itself was recovered in high yield from the di-THP derivative by stirring in methanol containing a catalytic amount of $p$-toluenesulphonic acid monohydrate at $25^{\circ} \mathrm{C}$ for several hours.

Considerable effort was now directed at finding the proper reaction conditions for replacing the $D$ moiety with less complex amides. Bell ${ }^{8}$ demonstrated that alkaline hydrolysis of compounds of type IVa and IVb or VIa and VIb leads exclusively to Va and Vb (Figure 6).

Therefore, since the product, $\mathrm{V}$, is the same regardless of which group is initially on $\mathrm{O}$ or $\mathrm{N}$, Bell proposed the following mechanism (using benzoyl acetyl reference compounds): (Figure 7).

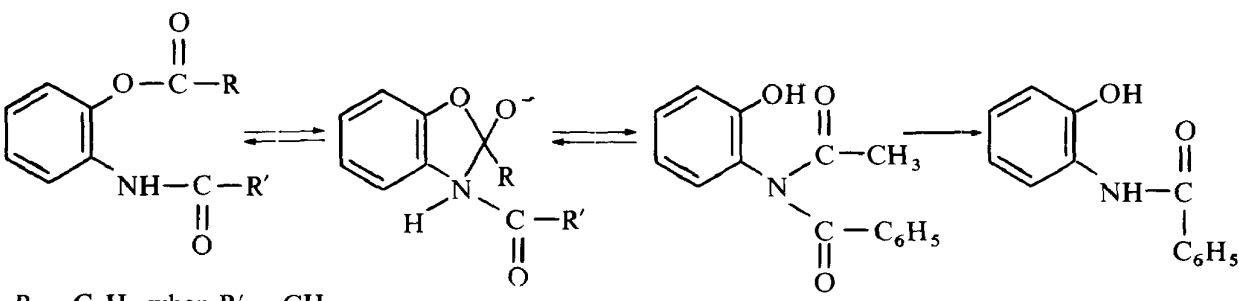

$\mathrm{R}=\mathrm{C}_{6} \mathrm{H}_{5}$ when $\mathrm{R}^{\prime}=\mathrm{CH}_{3}$ and

$\mathrm{R}=\mathrm{CH}_{3}$ when $\mathrm{R}^{\prime}=\mathrm{C}_{6} \mathrm{H}_{5}$

VII

Figure 7. (Reproduced by permission of Journal of Antibiotics, Tokyo)

The one acyl group of the intermediate VII (Figure 7) which is the more susceptible to nucleophilic displacement will be the one which is displaced to give the observed ortho-acylaminophenol. This particular reaction henceforth applied to the coumermycin sequence will be referred to as the transacylation reaction.

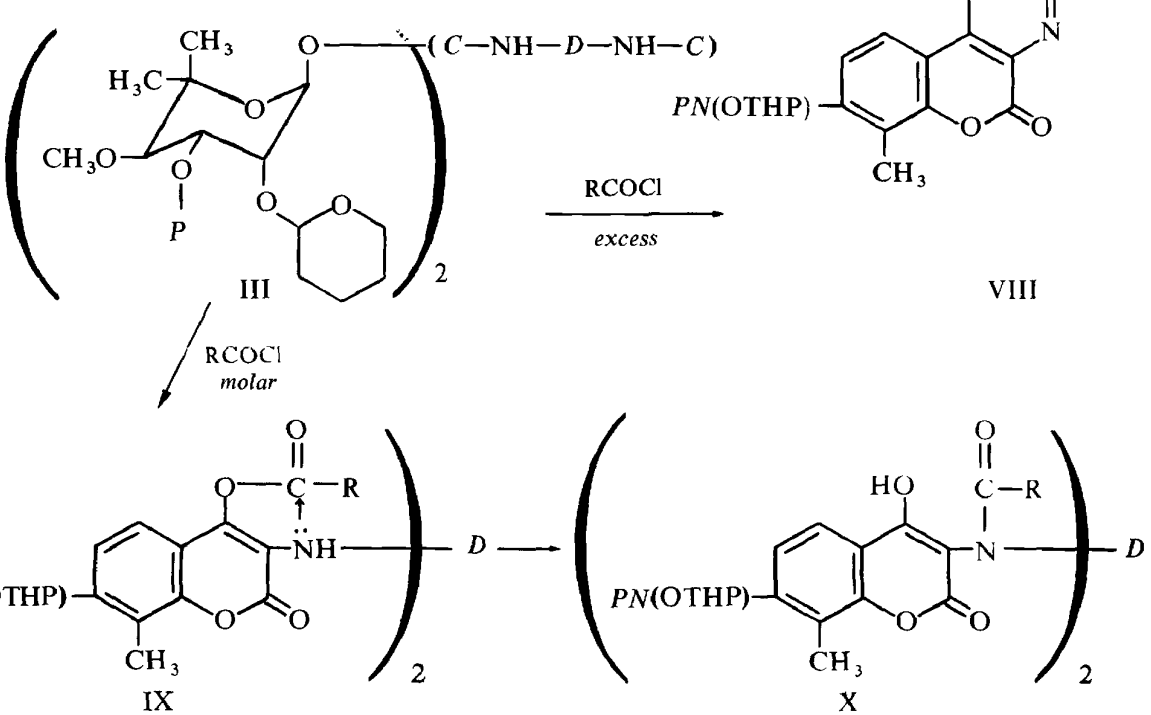

Figure 8. (Reproduced by permission of Journal of Antibiotics, Tokyo) 
When di-THP coumermycin $A_{1}$ (III) was reacted with acylating agents under rather vigorous conditions, e.g. under reflux conditions in pyridine using large molar excesses of acid halide or anhydride, oxazolocoumarins (Compound VIII of Figure 8) were found to be a major constituent of the product mixture ${ }^{6}$. The oxazolocoumarins VIII (Figure 8) were further extensively studied and subjected to a variety of hydrolytic conditions in an effort to open the oxazole ring.

The action of alkali upon coumarins is varied and dependent upon structure. Further, the extent of exposure to alkali can be an important factor as well. The initial action of alkali upon coumarins is always the opening of the pyrone ring with subsequent formation of either a coumarinic acid salt (Compound XI, cis configuration, of Figure 9) or the more stable coumaric acid salt (Compound XII, trans configuration).<smiles>O=c1ccc2ccccc2o1</smiles>

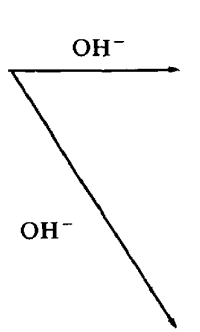<smiles>[N+]#[N+]Oc1ccccc1/C=C\C(=O)[O-]</smiles>

XI<smiles>[N+]Oc1ccccc1/C=C/C(=O)O</smiles>

XII

Figure 9

Normally, acidification of the reaction mixture regenerates the starting coumarin. However, in the case of the 4-hydroxy coumarins, this statement must be further clarified since the possibility of 2,4-coumarin/chromone tautomerism also exists (Compound XIII of Figure 10).<smiles>O=c1cc(O)c2ccccc2o1</smiles>

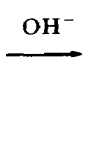<smiles>O=c1cc(O)oc2ccccc12</smiles> 
Alkaline hydrolysis of 7-acetoxy-8-methyl-2'-(methyl)-coumarino-(3:4)oxazole (Compound XIV of Figure 11) afforded structure XV.<smiles>COc1ccc2c(oc(=O)c3nc(C)oc32)c1CCOc1ccc2c(NC(C)=O)c(=O)oc3c(C)c(OC(C)=O)ccc3c1-2</smiles>

Figure 11

Attempts at ring closure of Compound XV (Figure 11) by acidification were unsuccessful and must be attributed, in part, to the stabilizing nature of the oxazole ring. Catalytic hydrogenation of the same oxazolocoumarin XIV in a 50/50 mixture of ethyl acetate/absolute ethanol using a platinum oxide/ 15 per cent acetic acid catalyst afforded 3-acetamido-4(H)-7-acetoxy-8methylcoumarin (Compound XVI of Figure 11).

Similar results were obtained from the alkaline hydrolyses studies on the $P N(\mathrm{OTHP})$ oxazolocoumarins as indicated in Figure 12 by the formation of Compound XVII from Compound VIII (Figure 8, $\mathrm{R}=\mathrm{CH}_{3}$ ).

Attempts at ring closure were again unsuccessful, and, as before, must be attributed primarily to the stabilizing influence of the oxazole ring.

Catalytic hydrogenation of the oxazolocoumarin VIII $\left(\mathrm{R}=\mathrm{CH}_{3}\right)$ using platinum oxide catalyst and the conditions indicated previously, afforded Compound XVIII (Figure 12), 3-acetamido-4(H)-8-methyl7-[2-O-tetrahydropyranyl-3-O-(5-methyl-2-pyrrolylcarbonyl)noviosyloxy]coumarin. Cleavage of the tetrahydropyranyl blocking group yielded 3-acetamido-4(H)-8-methyl-7-[3-O-(5-methyl-2-pyrrolylcarbonyl)noviosyloxy]coumarin. Compounds of this type were devoid of biological activity.

After our initial studies on the oxazolocoumarins, it was soon realized that treatment of di-THP coumermycin $A_{1}$ with the same acylating agents under milder conditions produced compounds which could be characterized as bis imides of ditetrahydropyranylcoumermycin $\mathrm{A}_{1}{ }^{6}$, Compound $\mathrm{X}$ in Figure 8. It seems most logical that this reaction occurs via the phenolic ester IX (Figure 8), and in all cases intermediate IX was considerably less stable than $\mathrm{X}$. 
J. G. KEIL et al.

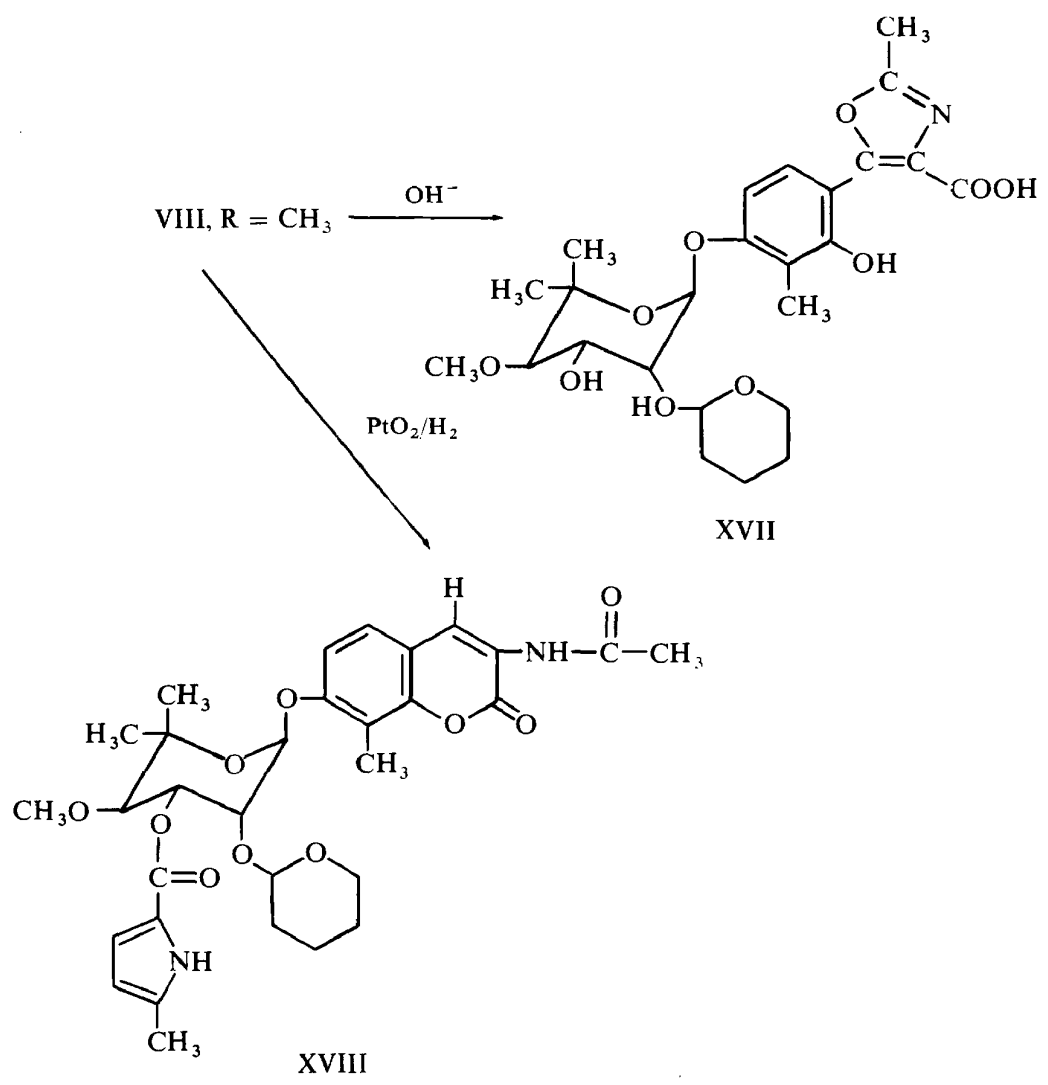

Figure 12

Cleavage of the bis imide $\mathrm{X}$ was best achieved by heating in fresh pyridine at about $50^{\circ} \mathrm{C}$ for $24-48$ hours. Although to some extent imide cleavage occurred during the acylation step in pyridine, it appeared to be substantially inhibited in many cases by the presence of pyridine hydrochloride. Consequently, the imide $X$ was isolated in many cases and recycled back into fresh pyridine. The imide cleavage product was always a mixture of the tetrahydropyranyl derivatives of the desired transacylation product (Compound XIX of Figure 13), a coumermycin $\mathrm{A}_{1}$ tetrahydropyranyl fragment (XX), representing cleavage of one of the $P N C$ - $\mathrm{NH}$ units, and ditetrahydropyranylcoumermycin $A_{1}$ (III) itself. The extent to which the desired product XIX is favoured in preference to III is dependent upon the nucleophilic character of the particular acyl group present and in line with the work of Bell $^{8}$ discussed earlier, the mechanism for this transacylation sequence must be clearly that detailed in Figure 8.

The imide mixture could be fractionated at this stage, and, indeed this was done in a number of cases to substantiate our reaction scheme. By far, however, the more practical approach was to remove the tetrahydropyranyl 
J. G. KEIL et al.

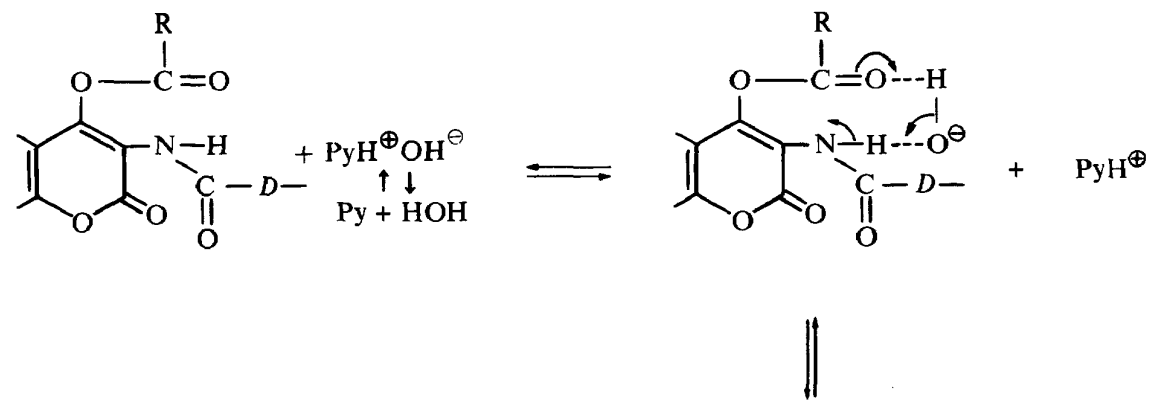<smiles>[R]C(=O)N(C(=O)O[PbH][PH2]O)c1c([O-])c(C)c(C)oc1=O</smiles>

Figure 14. (Reproduced by permission of Journal of Antibiotics, Tokyo)

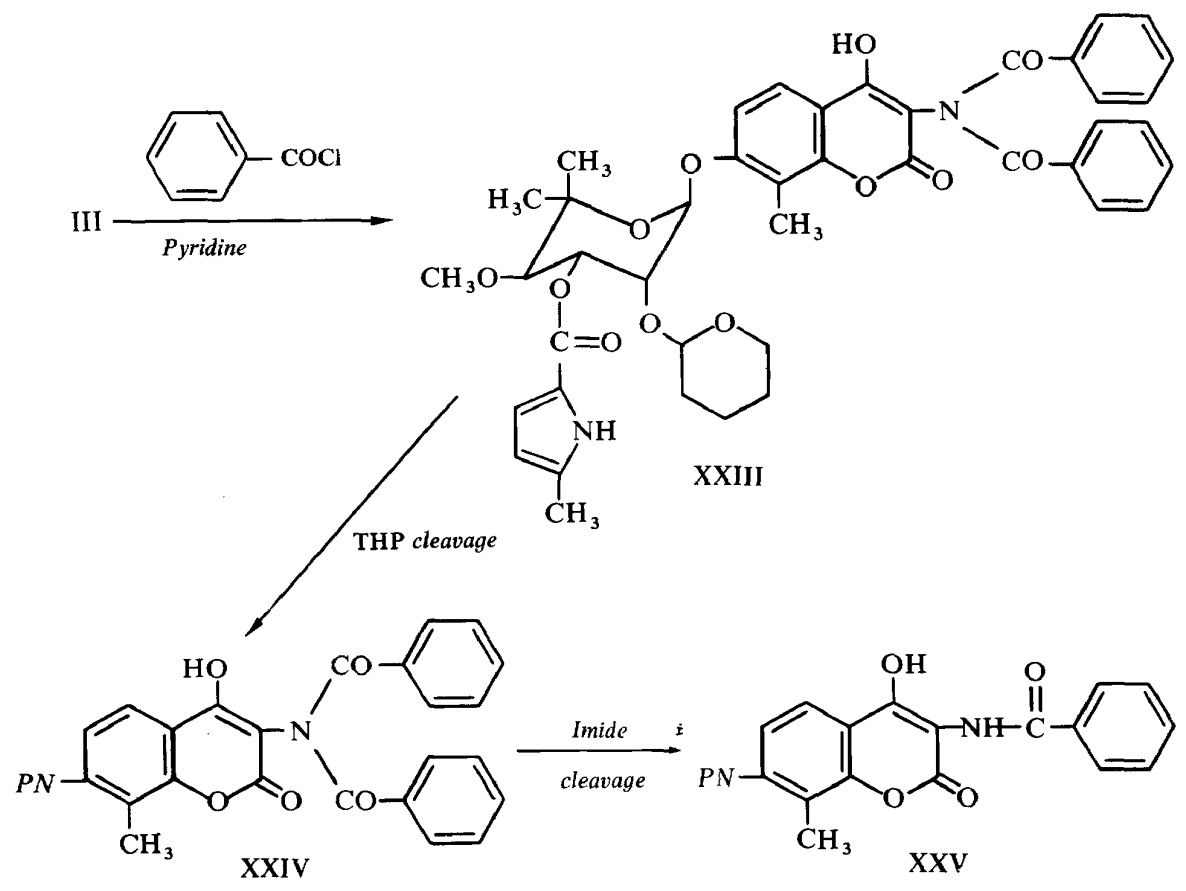

Figure 15 
Additional evidence supporting our transacylation mechanism was provided by the isolation of 3-dibenzoylamino-4-hydroxy-8-methyl-7-[2-Otetrahydropyranyl-3-O-(5-methyl-2-pyrrolylcarbonyl)noviosyloxy]coumarin (Compound XXIII of Figure 15) from a reaction mixture of ditetrahydropyranyl coumermycin $A_{1}$ (III) with an equimolar concentration of benzoyl chloride in pyridine under very mild conditions. Removal of the tetrahydropyranyl blocking group by methanol exchange as previously discussed afforded 3-dibenzoylamino-4-hydroxy-8-methyl-7-[3-O-(5-methyl2-pyrrolylcarbonyl)noviosyloxy]coumarin (Compound XXIV of Figure 15). This compound was comparable in all respects to the intact bis imides X. It had no amide-II (NH) band, and the ratio of aromatic to aliphatic protons in its integrated NMR spectrum was exactly as anticipated for PNCdibenzoylimide. The $N$-dibenzoylimide XXIV was readily cleaved in warm pyridine as previously described to give a high yield of 3-benzamido4-hydroxy-8-methyl-7-[3-O-(5-methyl-2-pyrrolyl carbonyl)noviosyloxy]coumarin $^{9}$ (Compound XXV of Figure 15). This compound was identical to the analogous derivative isolated from a previous transacylation mixture.

An alternative to the above discussed pyridine procedure, in which tetrahydropyranyl coumermycin $\mathrm{A}_{1}$ was. dissolved in tetrahydrofuran and treated at $0^{\circ}$ to $25^{\circ} \mathrm{C}$ sequentially with five molar equivalents of triethylamine and four molar equivalents of an acid chloride, was found to be beneficial in many cases. This procedure was particularly applicable to acid chlorides, which are at least as reactive as benzoyl chloride, while the pyridine procedure was preferred for less reactive acylating agents. In several cases the bis imide $\mathrm{X}$ (Figure 8 ) so produced was readily purified and completely characterized.

The transacylation reactions described above were useful for the preparation of a number of semisynthetic coumermycin antibiotics, many of which possessed physical and biological properties more favourable than coumermycin $\mathrm{A}_{1}$ itself. However, these reactions were found to be generally applicable only to acid chlorides and anhydrides. Since the mechanism of the transacylation reaction precludes the preparation of sulphonamide, ureide, thioureide, Schiff base and secondary amine derivatives at the coumarin-3-amine, derivatives of this type were best prepared by direct condensation with 3-amino-4-hydroxy-8-methyl-7-[3-O-(5-methyl-2pyrrolylcarbonyl)noviosyloxy]coumarin, 'PNC-amine' ${ }^{10}$. The preparation of this important intermediate is illustrated in Figure 16. Under carefully controlled conditions, the sodium salt of coumermycin $A_{1}$ reacts with benzyloxycarbonyl chloride (usually in tetrahydrofuran at $25^{\circ} \mathrm{C}$ adding the appropriate amount of triethylamine) to produce the bis carbamoyl imide XXVI which was characterized in the usual manner from its IR, NMR, and microchemical analyses. Apparently under these conditions, the 2'-hydroxyl of the noviose moieties is not sufficiently nucleophilic to attack benzyloxycarbonyl chloride to produce the carbonate ester. It would seem logical to presume in this case that transacylation also occurs via the mechanism suggested earlier in our discussion in view of the non-reactivity of ditetrahydropyranylcoumermycin $A_{1}$ with isocyanates, isothiocyanates, and alkyl and aryl sulphonyl chlorides.

Cleavage of the bis carbamoyl imide XXVI in warm pyridine 


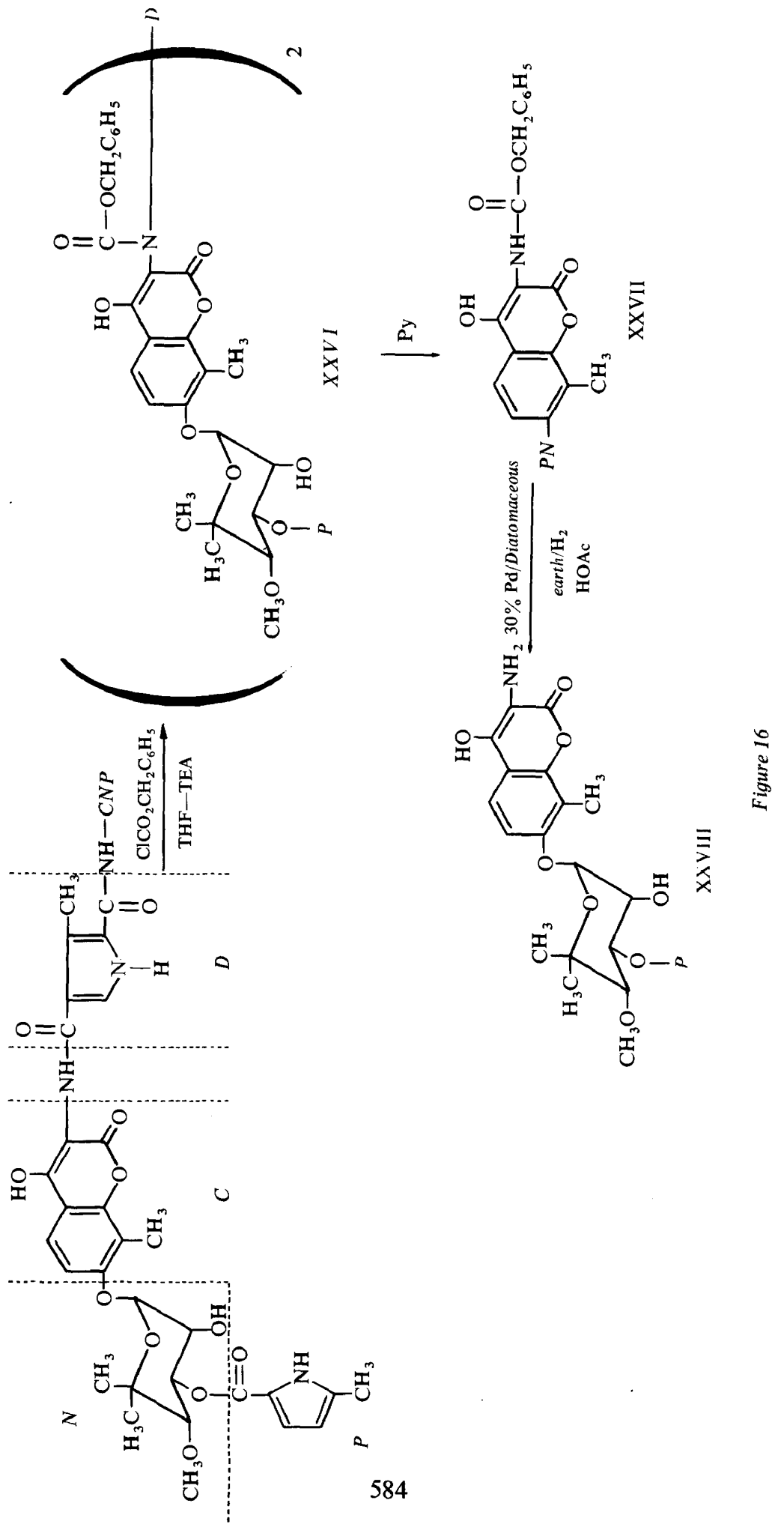


yielded 3-benzyloxycarbonylamido-4-hydroxy-8-methyl-7-[3-O-(5-methyl2-pyrrolylcarbonyl)noviosyloxy]coumarin (Compound XXVII of Figure 16) which was further cleaved by catalytic reduction (usually in glacial acetic acid using a 30 per cent palladium on diatomaceous earth catalyst) to afford PNC-amine (Compound XXVIII of Figure 16). Recrystallization from acetone and $n$-hexane afforded a light yellow-gold solid (melting point : $196-200^{\circ} \mathrm{C}$ ) whose IR and NMR spectra were consistent with the title compound. It was sensitive to oxidation, particularly when in solution.

\section{BIOLOGICAL EVALUATION OF SEMISYNTHETIC COUMERMYCINS}

One of the first compounds prepared by us was the benzamido derivative $6,11,12$. In view of its satisfactory properties of solubility, lack of irritation and good antibacterial activity, it was chosen as a standard for evaluation of other semisynthetic coumermycins. A comparison of the MIC's for this $P N C$-benzamido derivative relative to novobiocin and coumermycin $\mathrm{A}_{\mathbf{1}}$ is shown in Table 3.

Table 3. Comparison of minimal inhibitory concentrations of $P N C-\mathrm{NHCOC}_{6} \mathrm{H}_{5}$ and related antibiotics $^{\mathrm{a}}$

\begin{tabular}{|c|c|c|c|}
\hline \multirow[b]{2}{*}{ Organism } & \multicolumn{3}{|c|}{ Minimal inhibitory concn $(\mu \mathrm{g} / \mathrm{ml})$} \\
\hline & Coumermycin $\mathrm{A}_{1}$ & Novobiocin & $P N C-\mathrm{NHCOC}_{6} \mathrm{H}_{5}$ \\
\hline Bacillus cereus (Yale) & 6.2 & 6.2 & 25 \\
\hline Staphylococcus aureus Smith & 0.1 & 0.4 & 0.4 \\
\hline S. aureus M2614 & 0.1 & 0.8 & 0.8 \\
\hline S. aureus M1845 & 0.1 & 1.6 & 0.8 \\
\hline S. albus NOTC 7292 & 0.4 & 100 & 6.2 \\
\hline Diplococcus pneumoniae M1638 & 0.4 & 1.6 & 1.6 \\
\hline Streptococcus faecalis ATCC 8043 & 6.2 & 1.6 & 6.2 \\
\hline$S$. pyogenes Digonnet $\neq 7$ & 0.8 & 1.6 & 1.6 \\
\hline Klebsiella pneumoniae (Yale) & 12.5 & 25 & 50 \\
\hline
\end{tabular}

2 The minimal inhibitory concentrations were determined by the twofold agar-dilution method. In this procedure, antibiotics were incorporated into Trypticase Soy Agar (BBL) with $2 \%$ defibrinated sheep blood added. The minimal inhibitory concentrations were determined after $18 \mathrm{~h}$ of incubation at $37 \mathrm{C}$.

Further, the $\mathrm{CD}_{50}$ data from Staph. aureus Smith, Strep. pyogenes Digonnet \#7, and Diplococcus pneumoniae for the benzamido derivative, novobiocin and coumermycin $\mathrm{A}_{1}$ are shown in Table 4.

Table 4. Comparison of the $\mathrm{CD}_{50}$ of $P N C-\mathrm{NHCOC}_{6} \mathrm{H}_{5}$ with that of related antibiotics ${ }^{\mathrm{a}}$

\begin{tabular}{lccc}
\hline \multicolumn{1}{c}{ Organism } & \multicolumn{3}{c}{ Oral CD $_{\text {so }}(\mathrm{mg} / \mathrm{kg})$} \\
\cline { 2 - 4 } & Coumermycin $\mathrm{A}_{1}$ & Novobiocin & PNC- $^{-} \mathrm{NHCOC}_{6} \mathrm{H}_{5}$ \\
\hline Staphylococcus aureus Smith & 17 & 10 & 5.5 \\
Streptococcus pyogenes Digonnet \#7 & $>200$ & $>400$ & 110 \\
Diplococcus pneumoniae & 180 & $>100$ & 90 \\
\hline
\end{tabular}

a Mice were infected by intraperitoneal administration of a sufficient number of bacterial cells $(>100 \mathrm{LD}$ so $)$ to kill all nontreated mice within $48 \mathrm{~h}$. In the case of $S$. aureus Smith, the challenge dose was administered in $5 \%$ gastric mucin. The curative doses for $50 \%$ of the animals $\left(\mathrm{CD}_{50}\right)$ were calculated at $96 \mathrm{~h}$ postchallenge. 
J. G. KEIL et al.

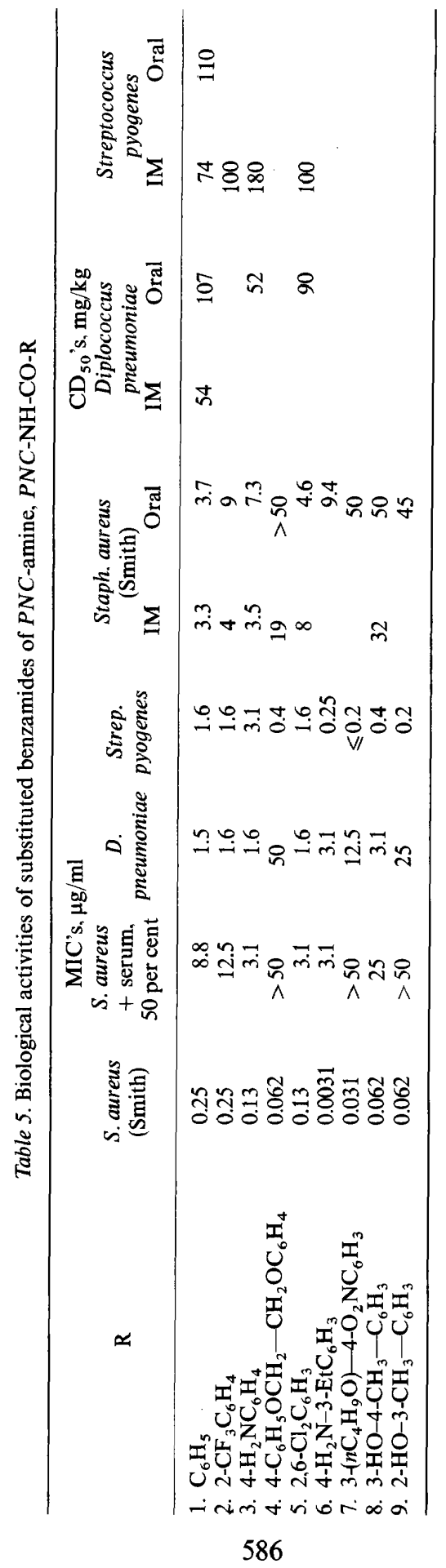


CHEMICALLY MODIFIED COUMERMYCIN

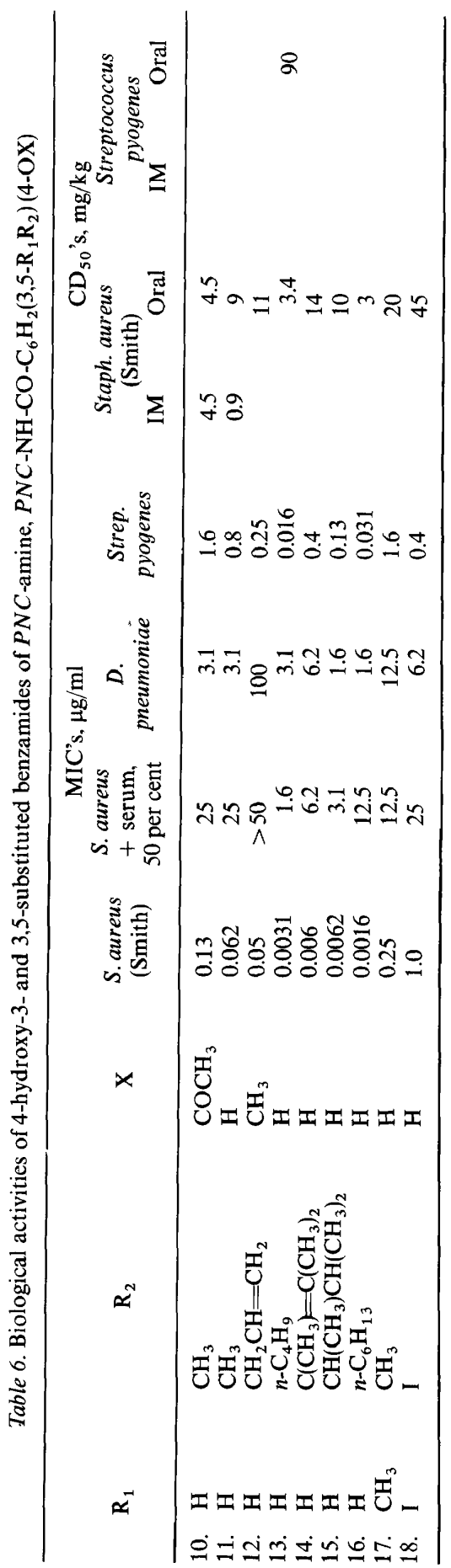


J. G. KEIL et al.

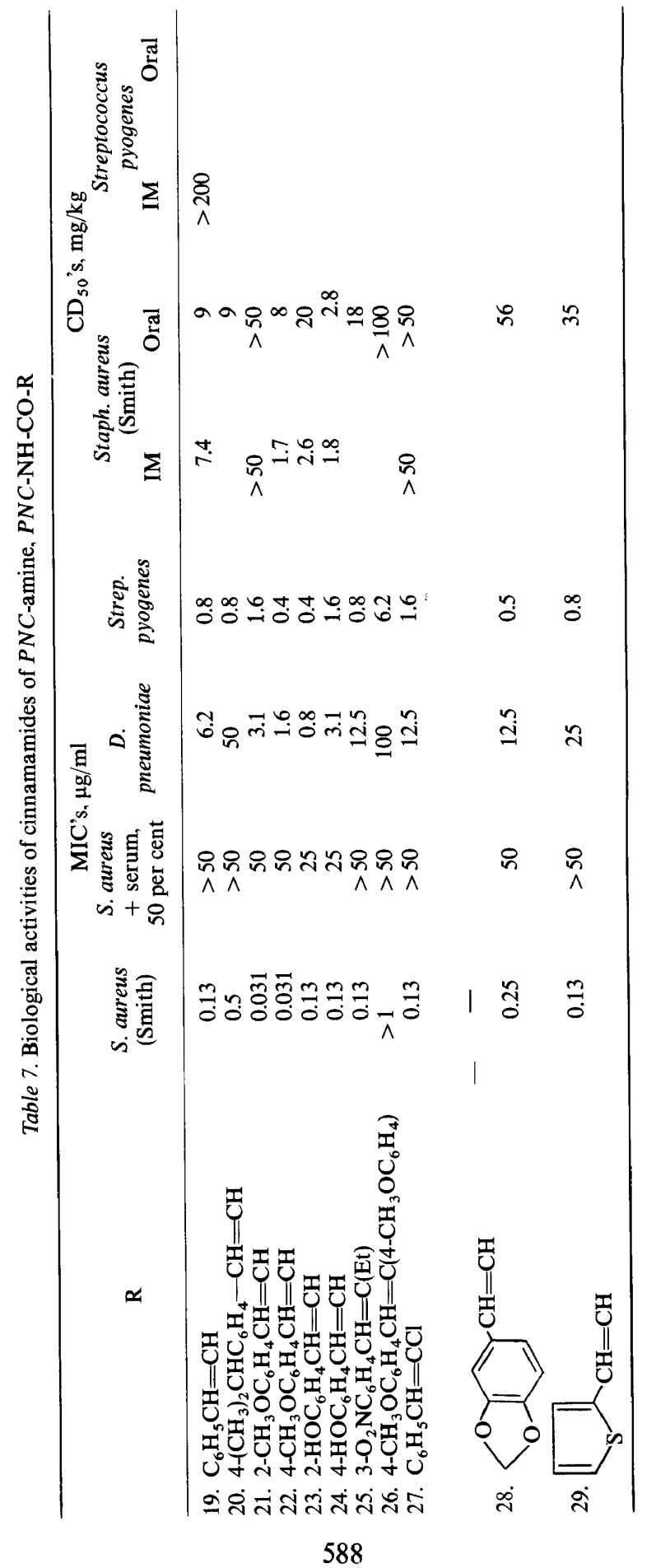




\section{CHEMICALLY MODIFIED COUMERMYCIN}

A number of substituted benzamides of $P N C$-amine are listed in Table $5^{6,11,12}$. The remarkable uniformity of activity of the compounds in this series is indicated by the fact that the compounds do not vary more than 4-fold from the standard benzamido derivative. Compound 4, the 4-phenoxy-ethoxybenzamido, Compound 7, the 3-n-butoxy-4nitrobenzamido, and Compound 9, the 2-hydroxy-3-methylbenzamido, are a few significant exceptions with respect to Diplococcus pneumoniae. An important contributing factor here appears to be the detrimental effect of a non-hydrophylic substituent meta to the amide linkage. The activity of the compounds indicated in Table 5 against streptococcal strains appears to be fairly consistent with perhaps Compound 7, the 3-n-butoxy-4-nitrobenzamido derivative being somewhat better. The most active antistaphylococcal compound indicated is the 3-ethyl-4-aminobenzamido derivative 6. The ratios of antimicrobial activity vs. Staphylococcus aureus in the presence and absence of serum indicates that a number of aromatic derivative 6. The ratios of antimicrobial activity versus Staphylococcus aureus Noteworthy examples in this regard are Compound 3, the 4-aminobenzamido, Compound 5, the 2,6-dichlorobenzamido, and Compound 6, the 3-ethyl-4aminobenzamido.

The compounds indicated in Table 6 show marked structural similarities to novobiocin ${ }^{11-14}$. Consistently high activity against streptococcus and staphylococcus is apparent in this series. The 3-allyl-4-methoxybenzamido derivative, Compound 12, indicates the importance of having the 4-oxygen function of the benzamide portion as a free hydroxyl. The relatively good activity of Compound 10, the 3-methyl-4-acetoxybenzamido, must in all probability be attributed to its facile hydrolysis, even in vitro. Compound 17 , the 3,5-dimethyl-4-hydroxybenzamido, and Compound 18, the 3,5-diiodo-4hydroxybenzamido, both illustrate the detrimental effect of two meta substituents. The most active compounds in this group are the $n$-butyl (13) and $n$-hexyl (16) derivatives both in vitro and in vivo. The effect of a properly lipophylic function in the meta position in conferring high in vitro activity is, unfortunately, offset by the high serum binding associated with this particular series.

Table 8. Biological activities of heteroaromatic derivatives of $P N C$-amine, $P N C$-N-CO-R

\begin{tabular}{|c|c|c|c|c|c|c|}
\hline \multirow{3}{*}{$\mathbf{R}$} & \multirow{3}{*}{$\begin{array}{l}\text { S. aureus } \\
\text { (Smith) }\end{array}$} & \multicolumn{2}{|c|}{ MIC's, $\mu \mathrm{g} / \mathrm{ml}$} & \multirow{3}{*}{$\begin{array}{c}\text { Strep. } \\
\text { pyogenes }\end{array}$} & \multirow{2}{*}{\multicolumn{2}{|c|}{$\begin{array}{c}\mathrm{CD}_{50} \text { 's, } \mathrm{mg} / \mathrm{kg} \\
\text { Staph. aureus } \\
\text { (Smith) }\end{array}$}} \\
\hline & & $\begin{array}{l}\text { S. aureus } \\
+ \text { serum }\end{array}$ & D. & & & \\
\hline & & 50 per cent & pneumoniae & & IM & Oral \\
\hline \multicolumn{7}{|l|}{ 30. $3^{\prime}-(2,6$-dichlorophenyl)-5'- } \\
\hline carboxamido & 0.5 & $>50$ & 50 & 0.2 & $>50$ & $>50$ \\
\hline \multicolumn{7}{|l|}{ 31. 3,5-dimethylisoxazolyl-4- } \\
\hline carboxamido & 1.0 & 50 & 25 & 3.1 & 5 & 24 \\
\hline \multicolumn{7}{|l|}{ 32. 5-chloro-2-thiophene } \\
\hline carboxamido & 0.062 & $>50$ & 6.2 & 0.8 & & 42 \\
\hline 33. 5-thiazolyl & 0.25 & 6.2 & 1.6 & 1.6 & 2.9 & 13 \\
\hline 34. 2-pyridyl & 1.0 & 25 & 3.1 & 1.6 & & 21 \\
\hline 35. 2-quinolyl & 0.1 & $>50$ & 25 & 1.0 & & $>100$ \\
\hline
\end{tabular}


J. G. KEIL et al.

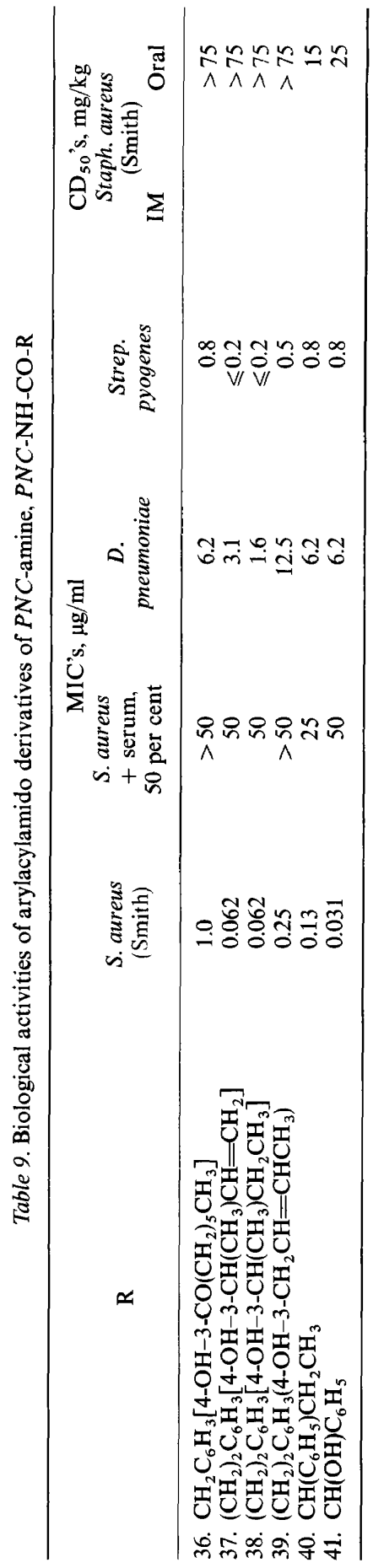




\section{CHEMICALLY MODIFIED COUMERMYCIN}

The remarkable similarity in activity of cinnamic acid derivatives to the benzamido series is indicated in Table $7^{6.11 .12}$. Although this would be expected on electronic grounds, it is rather surprising since cinnamamides are sterically different from benzamides.

A number of heteroaroyl derivatives are listed in Table $8^{6,11,12}$. On the whole, compounds in this category were very highly serum inactivated. The 5-thiazolyl compound (33) is the exception here having moderate in vitro and in vivo activity, and a fairly low degree of serum binding.

A series of arylacylamido derivatives are listed in Table $9^{10}$. Compounds 37 and 38, the 3-[3- $\alpha$-methylallyl-4-hydroxy]phenylpropionamido and 3-[3- $\alpha$-methylpropyl-4-hydroxy]phenylpropionamido, respectively, exhibit the best in vitro activity of the compounds listed. Compound 36, the 3 heptanoyl-4-hydroxyphenylacetamido, demonstrates the unfavourable effect of a keto function at position 3 on the ring. Compounds 40 , the 2-phenylbutyramido, and 41 , the $\alpha$-hydroxyphenylacetamido, illustrate the possible favourable effect of alpha substituents on both the in vitro and in vivo activity. Again, however, most compounds of this type were highly serum inactivated.

$P N C$-amine condensed readily with amino acids via the mixed anhydride or carbodiimide procedures. It also condensed readily with isocyanates to form ureides, with isothiocyanates to form thioureides, with alkyl halides to form secondary amines, with sulphonyl chlorides to form sulphonamides, or with aldehydes to form Schiff bases ${ }^{10.12}$. A few representative examples of the above type compounds are found in Table 10 in addition to the PNC.

Table 10. Biological activities of related derivatives of $P N C$-amine, $P N C$-NH-R

\begin{tabular}{|c|c|c|c|c|c|c|}
\hline \multirow{3}{*}{$\mathbf{R}$} & \multicolumn{4}{|c|}{ MIC's, $\mu \mathrm{g} / \mathrm{ml}$} & \multirow{2}{*}{\multicolumn{2}{|c|}{$\begin{array}{l}\mathrm{CD}_{50} \text { 's, } \mathrm{mg} / \mathrm{kg} \\
\text { Staph. aureus } \\
\text { (Smith) }\end{array}$}} \\
\hline & \multirow{2}{*}{$\begin{array}{l}\text { S. aureus } \\
\text { (Smith) }\end{array}$} & \multirow{2}{*}{$\begin{array}{l}\text { S. aureus } \\
+ \text { serum, } \\
50 \text { per cent }\end{array}$} & \multirow{2}{*}{$\begin{array}{c}\text { D. } \\
\text { pneumoniae }\end{array}$} & \multirow{2}{*}{$\begin{array}{c}\text { Strep. } \\
\text { pyogenes }\end{array}$} & & \\
\hline & & & & & $\mathrm{IM}$ & Oral \\
\hline 42. $\mathrm{COOCH}_{2} \mathrm{C}_{6} \mathrm{H}_{5}$ & 0.25 & 50 & 3.1 & 0.8 & 1 & $>50$ \\
\hline 43. $\mathrm{H}$ & $>1$ & $>50$ & 25 & 6.2 & & $>100$ \\
\hline 44. $\mathrm{COCH}_{2} \mathrm{CH}_{2} \mathrm{NH}$ & 0.5 & $>50$ & 25 & $>1$ & & $>75$ \\
\hline 45. $\mathrm{CONHCH}_{2} \mathrm{CH}_{3}$ & $>1$ & 50 & 6.2 & 1.6 & & $>100$ \\
\hline 46. $\mathrm{CONHC}_{6} \mathrm{H}_{5}$ & $>1$ & 12.5 & & 1.6 & & 25 \\
\hline 47. $\mathrm{CONHC}_{6} \mathrm{H}_{4} \mathrm{NH}_{2}-4$ & $>1$ & $>50$ & 12.5 & 3.1 & & \\
\hline 48. $\mathrm{SO}_{2} \mathrm{C}_{6} \mathrm{H}_{4} \mathrm{CH}_{3}-4$ & $>1$ & $>50$ & 25 & 0.8 & & $>100$ \\
\hline
\end{tabular}

carbobenzoxamido derivative (42) and $P N C$-amine itself (43). It is obvious that derivatives of this nature were of too low an order of activity to be of any practical value. The free amine exhibited low in vitro activity and failed to cure mouse infections. The $P N C$-carbobenzoxamido derivative (42) had rather significant in vitro activity against Diplococcus pneumoniae, Streptococcus pyogenes and three strains of Staphylococcus aureus. Further, its in vivo activity against $S$. aureus Smith (mice) via the intramuscular route was significant, although it had no activity orally.

A number of fatty acid-type, simple unsaturated and branched aliphatic derivatives of $P N C$-amine are listed in Table $11^{10,12}$. The activity maximum with respect to derivatives of the fatty acid-type appears to be reached at 
J. G. KEIL et al.

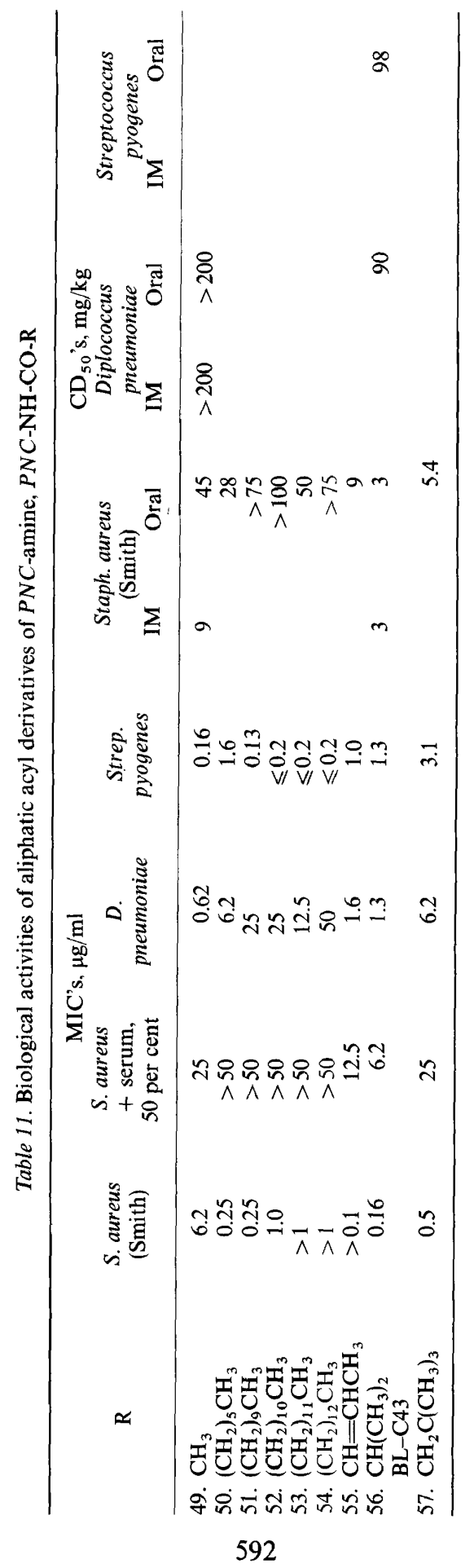




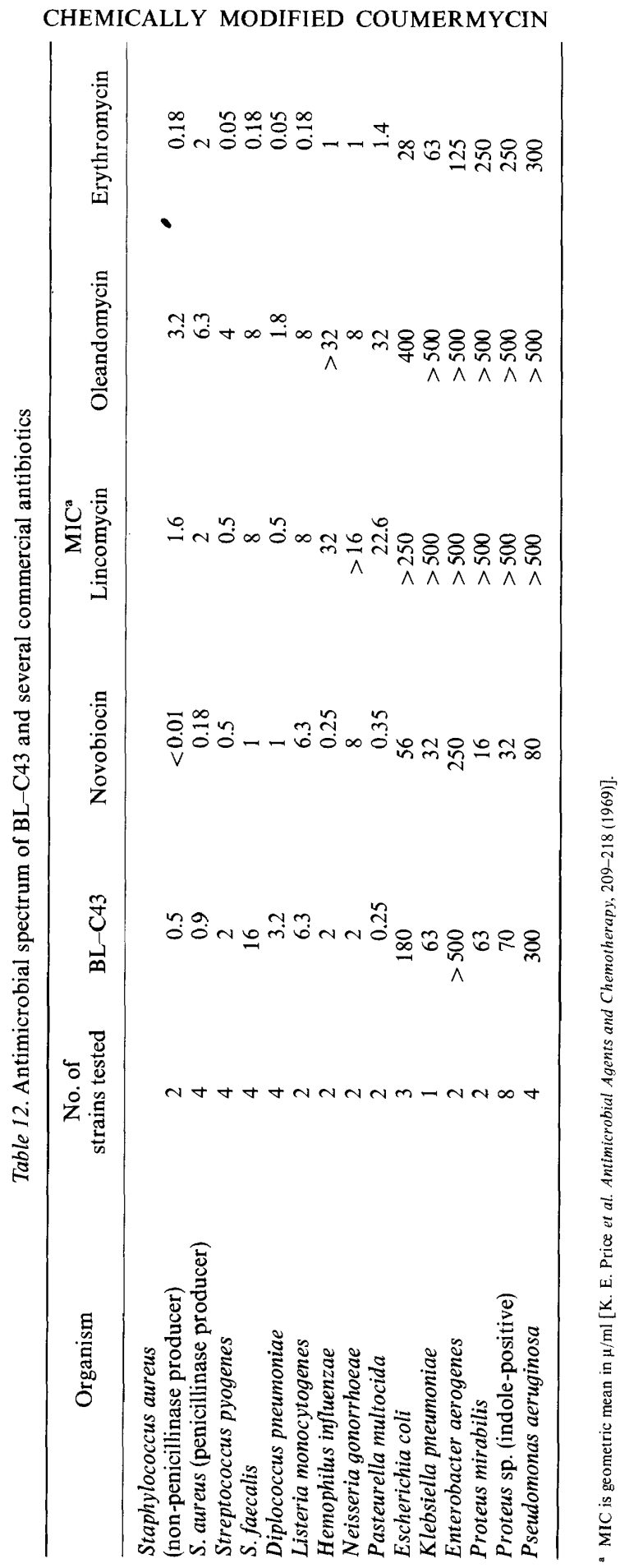


13 carbons, i.e. the tridecanamido derivative 53. Compounds of this nature were very highly serum inactivated. The simple unsaturated Compound 55, the 2-butenamido, has appreciable in vitro activity and in vivo activity (oral $\mathrm{CD}_{50}$ of $9 \mathrm{mg} / \mathrm{kg}$ versus Staphylococcus aureus Smith) as does Compound 57, the tert-butylacetamido derivative (oral $\mathrm{CD}_{50}$ of $5.4 \mathrm{mg} / \mathrm{kg}$ versus Staphylococcus aureus Smith).

As indicated at the beginning of our discussion, the objectives of this structural modification programme were directed primarily at maintaining or enhancing the desirable properties of coumermycin $A_{1}$ and reducing or eliminating some of its undesirable characteristics. A certain degree of success has been achieved in this regard. A number of the new semisynthetic antibiotics discussed above possessed markedly greater oral absorbability in mice. This improvement in some cases was $>25$-fold over the parent compound and more than compensated for the lower antibacterial potency possessed by most of the derivatives. Further, some of the more active compounds showed a significant improvement in the in vivo antistaphylococcal therapeutic effect and marked improvements in oral efficacy in experimental D. pneumoniae and Streptococcus pyogenes mouse infections.

The compounds having the most improved activity against gram-positive bacteria were produced when the 3-amino group of the coumarin moiety was acylated with aromatic or heteroaromatic carboxylic acids, either with or without mono- or disubstituents on the ring ${ }^{15}$. Although a number of compounds prepared from branched-chain aliphatic acids had comparable in vivo efficacy, those derivatives derived from $n$-aliphatic or aryl-substituted aliphatic acids, including those from trisubstituted benzoic acids, were considerably less effective.

Compounds demonstrating the broadest spectrum of antibacterial activity were members of the 3-substituted-4-hydroxybenzoic acid class. The most active representative of this class was the 3-n-hexyl-4-hydroxy benzoic acid derivative. It had comparable efficacy to coumermycin $A_{1}$ when administered parenterally in experimental $K$. pneumoniae mouse infections but, unfortunately, it proved to be ineffective when given by the oral route.

Among the aliphatic derivatives, Compound 56, the isobutyramido (code number BL-C43), was by far the most interesting. This derivative was not only characterized by its overall trend toward improved in vitro activity, but also by its significant in vivo activity.

On a comparative basis with other semisynthetic coumermycins, the finding of 78 per cent serum binding of BL-C43 by 95 per cent human serum was deemed highly significant and in all probability accounts for its effectiveness in the standard mouse infection. All other interesting derivatives were found to be highly serum bound, generally in the order of 95 to 99 per cent. In view of this information, further efforts in this programme were now directed toward a complete evaluation of BL-C43 itself. The balance of this discussion consequently will deal with the preclinical and clinical data obtained on BL-C43.

The in vitro antibacterial spectra of BL-C43 relative to several other antibiotics are shown in Table 12.

On the whole BL-C43 is of interest primarily because of its gram-positive spectrum, but it does show significant activity against the gram-negative 
organisms Hemophilus influenzae, Neisseria gonorrhoeae and Pasteurella multocida.

The rate of resistance development by Staphylococcus aureus A9497 to BL-C43, erythromycin and lincomycin was determined by multiple transfers on Heart Infusion Agar plates containing the antibiotics ${ }^{16}$. Stepwise development of resistance was closely patterned in all cases with all three antibiotics showing comparable rates of resistance development. In one particular experiment, although considerable cross-resistance was observed between lincomycin, erythromycin and oleandomycin, no such cross-resistance was observed between BL-C43 and these three antibiotics.

The effect of inoculum size on the MIC of BL-C43 and of the other antibiotics for S. aureus A9537 is shown in Table 13. As can be seen, a 10000fold increase in the concentration of $S$. aureus only doubled the concentration of $\mathrm{BL}-\mathrm{C} 43$ required to stop the growth of the organism.

Table 13. Effect of inoculum size on the activity of BL-C43 and several other antibiotics against Staphylococcus aureus A9537

\begin{tabular}{llll}
\hline \multicolumn{1}{c}{ Antibiotic } & $\begin{array}{c}\text { Dilution of an } 18-\mathrm{h} \text { culture } \\
10^{-6}\end{array}$ & & of S. aureus A9537 \\
& & 0.2 & $10^{-2}$ \\
\hline BL-C43 & $0.2^{\mathrm{b}}$ & 0.05 & 0.4 \\
Novobiocin & 0.025 & 6.3 & 0.05 \\
Oleandomycin & 3.1 & 1.6 & 12.5 \\
Lincomycin & 0.8 & 0.4 & 1.6 \\
Erythromycin & 0.2 & 0.8 \\
\hline
\end{tabular}

2 Contained $4.5 \times 10^{8}$ viable cells $/ \mathrm{mi}$

${ }^{b}$ Minimal inhibitory concentration in micrograms per millilitre[K. E. Price et al. Antimicrobial Agents and Chemotherapy 209-218 (1969)].

A comparative study of the relative bacteriostatic and bactericidal concentrations of BL-C43 against two strains of staphylococci, one of which was a penicillinase-producing organism (A9606), indicated that BL-C43 was bactericidal for both cultures at a concentration only 2- to 4-fold higher than the MIC.

BL-C43 was found to be remarkably stable to low $\mathrm{pH}^{16}$. The time required for destruction of one-half of an original $100 \mu \mathrm{g} / \mathrm{ml}$ concentration at $\mathrm{pH} 2.0$ was found to be in excess of 10 hours. This would imply that the compound is not significantly degraded upon exposure to the highly acidic gastric juices following its oral administration.

A comparison of the relative effectiveness of BL-C43 to that of coumermycin $A_{1}$ and other antibiotics in experimental mouse infections is shown in Table 14. BL-C43 demonstrated marked improvements in activity versus staphylococcal strains as compared to novobiocin, lincomycin and oleandomycin. Further, it was far superior in all cases against $S$. pyogenes and D. pneumoniae.

The surprisingly high efficacy of BL-C43 must be attributed in part to its good oral absorption by mouse, dog and human. A comparison of the 
J. G. KEIL et al.

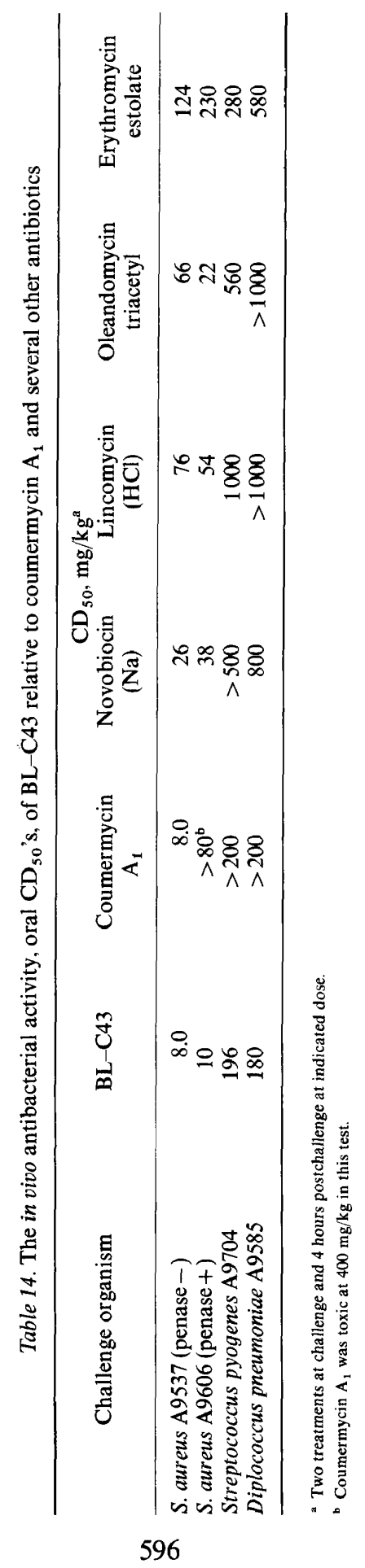


concentrations of BL-C43 in the blood of mice after oral administration of a $25 \mathrm{mg} / \mathrm{kg}$ dose relative to coumermycin $\mathrm{A}_{1}$ and several other antibiotics is shown in Figure 17.

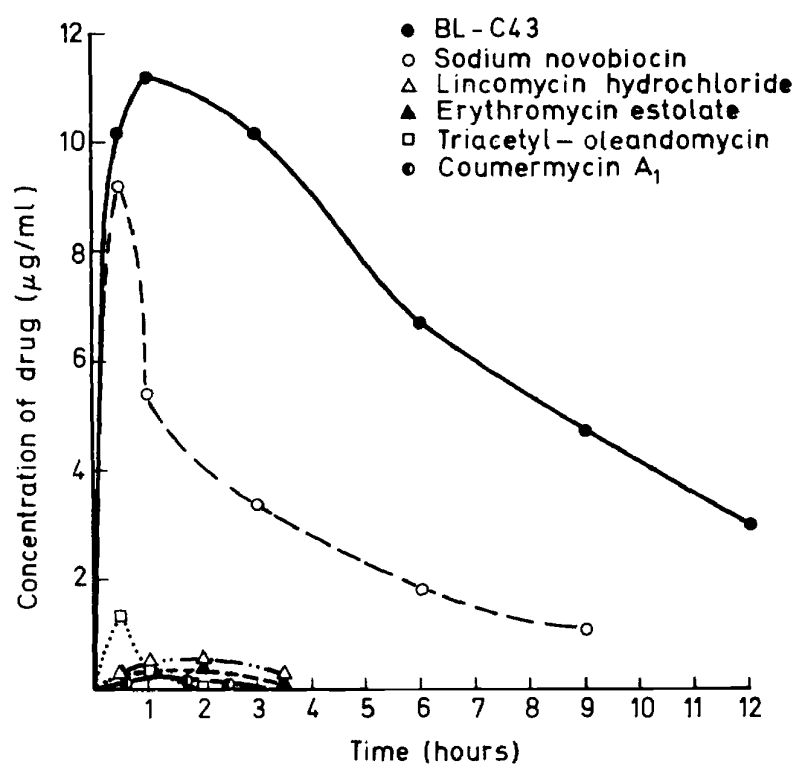

Figure 17. Concentrations of BL-C43, coumermycin $\mathrm{A}_{1}$, and several other drugs in the blood of mice after oral administration of a $25 \mathrm{mg} / \mathrm{kg}$ dose

Peak blood levels for BL-C43 were reached one hour postadministration at a concentration in excess of $11 \mu \mathrm{g} / \mathrm{ml}$. Further, the compound persisted in the blood for a long period of time with levels of $3 \mu \mathrm{g} / \mathrm{ml}$ being observed after 12 hours. Novobiocin also peaked rapidly at about $9 \mu \mathrm{g} / \mathrm{ml}$ and persisted for approximately 9 hours. The superior absorption of BL-C43 to that of coumermycin $A_{1}$ is obvious. Similar results were obtained after oral dosing of BL-C43 in the beagle dog, as indicated in Figure 18.

Peak concentrations of BL-C43 in the blood of mice after IM administration were found to be approximately $30 \mu \mathrm{g} / \mathrm{ml}$ after one hour, about double that for either novobiocin or lincomycin.

Cumulative evidence has demonstrated that the primary route of elimination of BL-C43 is via biliary excretion. Table 15 shows the concentration and percentage recovery of BL-C43 in urine samples collected up to 4.5 hours after dosing.

These results indicate that less than 1 per cent of the dose administered was recovered in the urine. Further, there was no evidence that the kidney, heart or lung tissues concentrate the drug. The antibiotic is in all probability accumulated by the liver, concentrated in the bile and subsequently released into the small intestine where it is presumably reabsorbed to a high degree. 


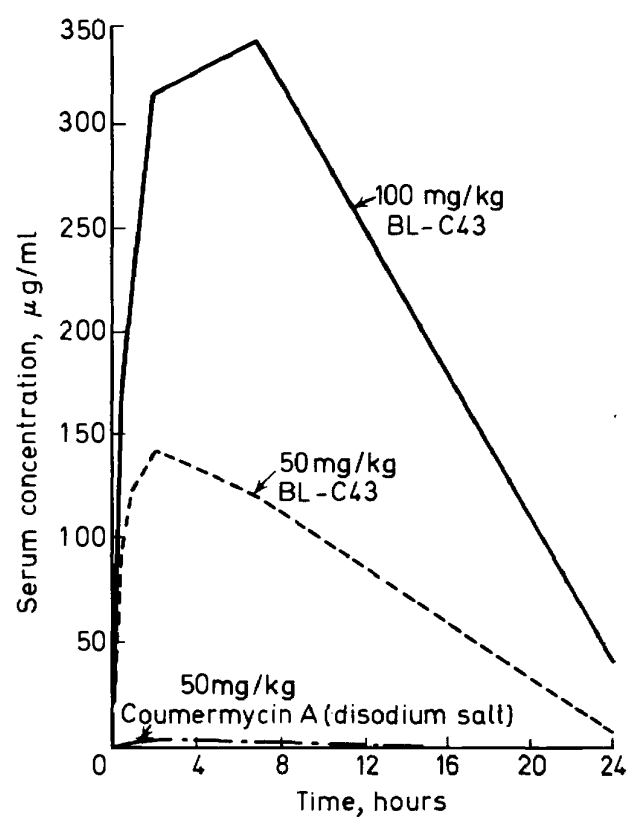

Figure 18. Blood (serum) levels of BL-C43 (Na) after single oral doses in dog

A comparison of the irritation liability of BL-C43, coumermycin $A_{1}$, and several other semisynthetic derivatives, was determined by measuring the extent of their irritating properties for the rat foot pad when injected as 1 and 5 per cent solutions or suspensions, the extent of oedema being measured by volumetric displacement. These results are shown in Figure 19. Coumermycin $\mathrm{A}_{1}$ proved extremely irritating at both concentrations. BL-C43 proved to be somewhat less irritating than coumermycin $A_{1}$. However, at the 24-hour measurement, BL-C43 could not be considered

Table 15. Urinary excretion of BL-C43 after intravenous administration of a $20 \mathrm{mg} / \mathrm{kg}$ dose to mice

\begin{tabular}{ccc}
\hline $\begin{array}{c}\text { Sampling interval } \\
(\mathrm{h})\end{array}$ & $\begin{array}{c}\mathrm{BL}-\mathrm{C} 43 \text { concentration } \\
(\mu \mathrm{g} / \mathrm{ml})\end{array}$ & $\begin{array}{c}\text { Percentage of administered } \\
\text { drug recovered }\end{array}$ \\
\hline $0.0-1.0$ & 9.1 & 0.11 \\
$1.0-1.5$ & 7.8 & 0.17 \\
$1.5-2.0$ & 3.4 & 0.08 \\
$2.0-2.5$ & 2.7 & 0.09 \\
$2.5-3.0$ & 2.7 & 0.03 \\
$3.0-3.5$ & 1.7 & 0.05 \\
$3.5-4.0$ & 2.0 & 0.04 \\
$4.0-4.5$ & $<1.5$ & - \\
Total & & 0.57 \\
\hline
\end{tabular}

[K. E. Price et al. An̨timicrobial Agents and Chemotherapy, 209-218 (1969)]. 


\section{CHEMICALLY MODIFIED COUMERMYCIN}

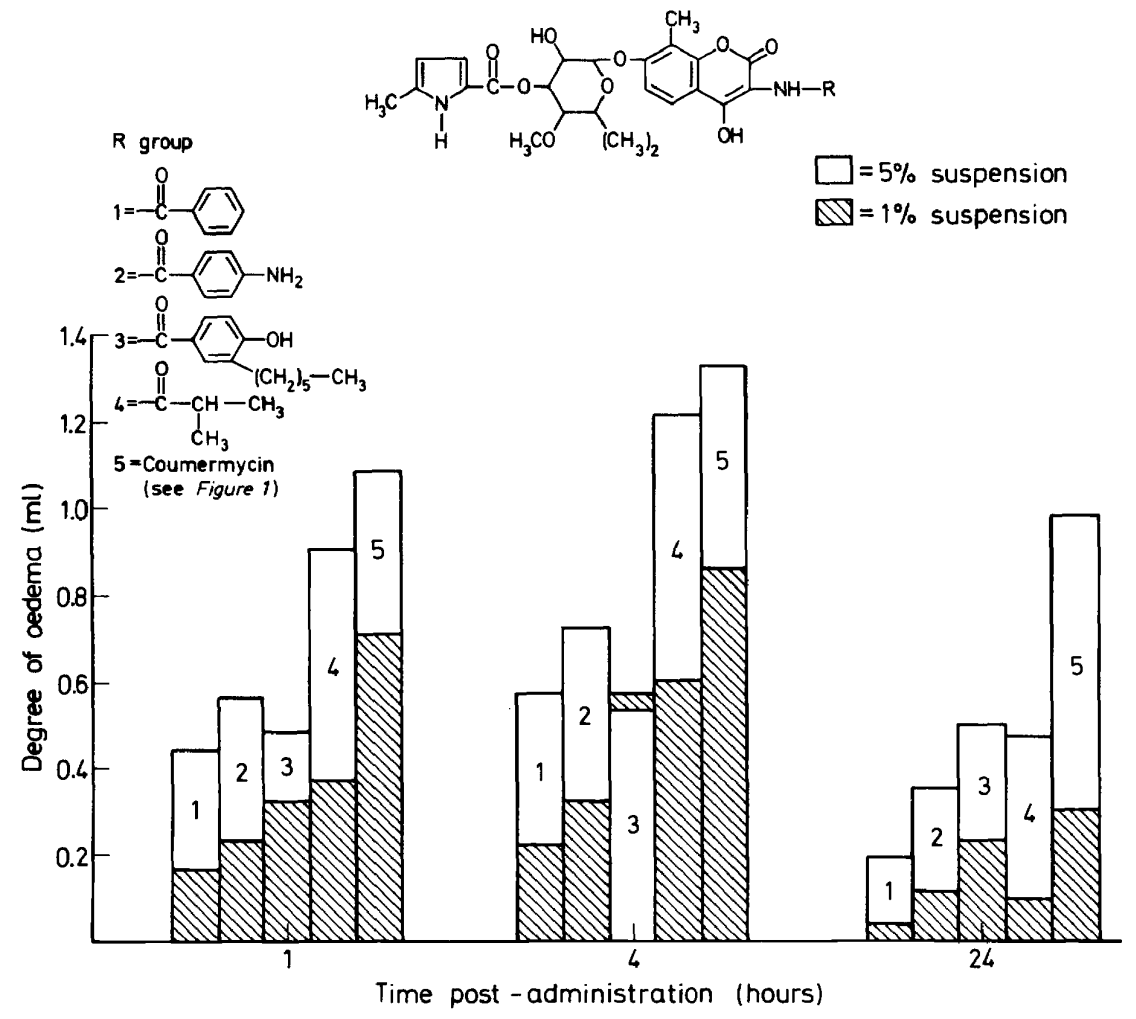

Figure 19. Irritation liability to the rat foot pad of BL-C43, coumermycin $\mathrm{A}_{1}$, and other coumermycin derivatives measured by volume displacement (K. E. Price et al., Appl. Microbiol. 19: 14-26)

irritating at all. The 3-n-hexyl-4-hydroxybenzoyl analogue (Compound 3) was only slightly more irritating at the 5 than at the 1 per cent level, due in all probability to its extremely low degree of solubility.

A comparison of the IP and PO acute $\mathrm{LD}_{50}$ values for BL-C43 in the mouse relative to coumermycin $A_{1}$ is shown in Table 16 . The marked decrease in the oral toxicity of BL-C43 is all the more surprising in view of its significantly higher degree of absorbability by this route than is coumermycin $\mathbf{A}_{1}$.

Table 16. Acute toxicity of BL-C43 and coumermycin $\mathrm{A}_{1}$

for mice

\begin{tabular}{lcc}
\hline \multicolumn{1}{c}{ Compound } & \multicolumn{2}{c}{ Route of administration } \\
& Intraperitoneal & Oral \\
\hline Coumermycin $\mathrm{A}_{1}$ & $230^{\mathrm{a}}$ & 2400 \\
BL-C43 & 350 & 1400 \\
\hline
\end{tabular}

- $\mathrm{LD}_{50}$ (lethal dose, 50 per cent) in $\mathrm{mg} / \mathbf{k g}$. 


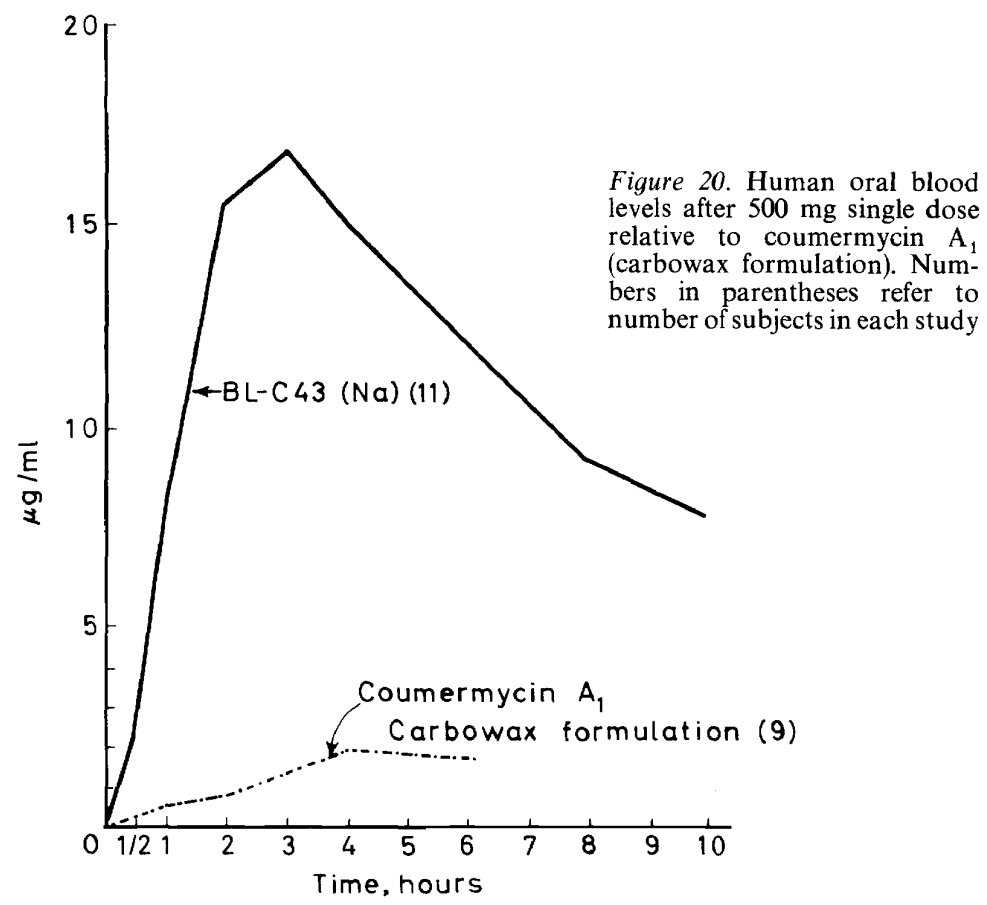

After completion of the extensive toxicological study of BL-C43 in rats and dogs, single-dose oral blood levels were carried out in human subjects. Again, as was the case in our animal studies, BL-C43 was found to be very well absorbed in humans. This fact is very clearly indicated in Figure 20 where the human oral blood level curve after a $500 \mathrm{mg}$ single dose of BL-C43 (Na) is compared to a carbowax formulation of coumermycin $A_{1}$ under the same conditions. Studies utilizing the mono- and di-sodium salts of coumermycin $A_{1}$ consistently gave blood levels of less than $0.1 \mu \mathrm{g} / \mathrm{ml}$ after a $500 \mathrm{mg}$ oral dose. It can be seen that BL-C43 reached a peak blood level in excess of $15 \mu \mathrm{g} / \mathrm{ml}$ after approximately 3 hours and was still at a high level after 10 hours (approximately $8 \mu \mathrm{g} / \mathrm{ml}$ ).

A comparison of the degree of serum binding to human serum of BL-C43 and coumermycin $A_{1}$, determined under standard conditions, is shown in Table 17. It is evident from these data that the low degree of serum binding of BL-C43 (78 per cent) must account for its outstanding efficacy in experimental infections.

However, in spite of the favourable evidence obtained from our extensive animal studies indicating the innocuous nature of BL-C43, the Phase I

Table 17. Serum binding data

\begin{tabular}{lcc}
\hline \multicolumn{1}{c}{ Antibiotic } & Per cent bound & Fraction free \\
\hline BL-C43 & 78 & 0.22 \\
Coumermycin $\mathrm{A}_{1}$ & 99 & 0.01 \\
\hline
\end{tabular}




\section{CHEMICALLY MODIFIED COUMERMYCIN}

clinical study of BL-C43 revealed a limiting toxicity of this antibiotic after 10 days of dosing at only $1 / 10$ to $1 / 5$ of the highest dose $/ \mathrm{kg}$ used in our toxicology study in dogs.

\section{ACKNOWLEDGEMENTS}

Due mention should be made of the following members of the Biochemical Research Department at Bristol Laboratories who made major contributions to the chemistry reported here: R. L. DeVault $\uparrow$, R. M. Downing, O. B. Fardig, C. M. Kalinowski, E. R. May, C. D. McDonnell $\ddagger$,F. A. O’Herron, E. A. Ragan, M. A. Rousche, R. H. Schreiber, C. L. Swanson $\ddagger^{2}$, A. L. Vulcano and D. F. Whitehead.

The authors also wish to acknowledge Drs H. Madissoo and V. Kadar of the Toxicology Department, and Dr M. E. Bierwagen of the Pharmacology Department, Bristol Laboratories, for the data on antibiotic blood levels in dogs and humans, respectively. We thank Dr James Taggart for the Phase I clinical data obtained on BL-C43.

Gratitude is expressed for permission to reproduce figures previously published elsewhere; in particular, Figures $1,3,6,7,8,13$ and 14 appeared in 'Semisynthetic coumermycins I. Preparation of 3-acylamido-4-hydroxy-8methyl-7-3-O-(5-methyl-2-pyrrolylcarbonyl) noviosyloxy coumarins' in the Journal of Antibiotics, Tokyo, 21, 551-566 (1968).

\section{REFERENCES}

1 H. Kawaguchi, M. Okanishi and T. Miyaki, US Pat. No. 3201386 (17 August 1965).

2 H. Kawaguchi, H. Tsukiura, M. Okanishi, T. Miyaki, T. Ohmori, K. Fujisawa and H. Koshiyama. J. Antibiotics (Tokyo) Ser. A 18, 1 (1965).

3 J. Berger, A. J. Schocher, A. D. Batcho, B. Pecherer, O. Keller, J. Maricq, A. E. Karr, B. P. Vaterlaus, A. Furlenmeier and H. Speigelberg. Antimicrobial Agents and Chemotherapy 778 (1965).

4 R. B. Walton, L. E. McDaniel and H. B. Woodruff. Develop. Ind. Microbiol. 3, 370 (1962).

5 J. W. Hinman, H. Hoeksema, E. L. Caron and W. G. G. Jackson. J. Am. Chem. Soc. 78, 1072 (1956).

6 J. G. Keil, I. R. Hooper, M. J. Cron, O. B. Fardig, D. E. Nettleton, F. A. O’Herron, E. A. Ragan, H. Schmitz, R. H. Schreiber and J. C. Godfrey. J. Antibiotics (Tokyo) 21, 551 (1968).

7 D. E. Nettleton. US Pat. No. 380994 (30 April 1968).

8 F. Bell, J. Chem. Soc. 2962 (1931).

9 J. G. Keil and I. R. Hooper. US Pat. No. 3428693 (18 February 1969).

10 J. G. Keil, I. R. Hooper, R. H. Schreiber, C. L. Swanson and J. C. Godfrey. Antimicrobial Agents and Chemotherapy 200 (1969).

11 J. G. Keil, I. R. Hooper, M. J. Cron, H. Schmitz, D. E. Nettleton and J. C. Godfrey. Antimicrobial Agents and Chemotherapy 120 (1968).

12 M. J. Cron, J. C. Godfrey, I. R. Hooper, J. G. Keil, D. E. Nettleton, K. E. Price and H. Schmitz. The Proceedings of the Sixth International Congress of Chemotherapy Vol. 2, p 1069 (1970).

13 H. Schmitz, R. L. DeVault, C. D. McDonnell and J. C. Godfrey. J. Antibiotics (Tokyo) 21, 603 (1968).

14 H. Schmitz and J. C. Godfrey. J. Antibiotics (Tokyo) 23, 497 (1970).

15 K.E. Price, D. R. Chisholm, J. C. Godfrey, M. Misiek and A. Gourevitch. Appl. Microbiol. 19, 14 (1970).

16 K. E. Price, D. R. Chisholm. F. Leitner and M. Misiek, Antimicrobial Agents and Chemother apy 209 (1969).

† Present address: Biochemical Research, Abbott Laboratories, North Chicago, Ill. 60064.

$\ddagger$ Former employees. 